\title{
The transcriptomic signature of fasting murine liver
} Milka Sokolović ${ }^{1}, 2$, Aleksandar Sokolović ${ }^{1}$, Diederik Wehkamp ${ }^{3}$, Emiel Ver Loren van Themaat ${ }^{3}$, Dirk R de Waart ${ }^{1}$, Lisa A Gilhuijs-Pederson ${ }^{3}$, Yuri Nikolsky ${ }^{4}$, Antoine HC van Kampen ${ }^{3}$, Theodorus BM Hakvoort ${ }^{1}$ and Wouter H Lamers*1

Address: ${ }^{1}$ AMC Liver Center, Academic Medical Center, University of Amsterdam, The Netherlands, ${ }^{2}$ Department of Medical Biochemistry, Academic Medical Center, University of Amsterdam, The Netherlands, ${ }^{3}$ Bioinformatics Laboratory, Academic Medical Center, University of Amsterdam, The Netherlands and ${ }^{4}$ GeneGo, Inc., St. Joseph, MI, USA

Email: Milka Sokolović - m.sokolovic@amc.uva.nl; Aleksandar Sokolović - a.sokolovic@amc.uva.nl; Diederik Wehkamp - d.wehkamp@gmail.com; Emiel Ver Loren van Themaat - p.e.verlorenvanthemaat@amc.uva.nl; Dirk R de Waart - d.r.dewaart@amc.uva.nl; Lisa A Gilhuijs-Pederson - l.a.pederson@amc.uva.nl; Yuri Nikolsky - yuri@genego.com; Antoine HC van Kampen - a.h.vankampen@amc.uva.nl; Theodorus BM Hakvoort - t.hakvoort@amc.uva.nl; Wouter H Lamers* - w.h.lamers@amc.uva.nl

* Corresponding author

Published: 6 November 2008

BMC Genomics 2008, 9:528 doi:10.1/86/147|-2164-9-528
Received: 25 March 2008

Accepted: 6 November 2008

This article is available from: http://www.biomedcentral.com/I47I-2/64/9/528

(c) 2008 Sokolović et al; licensee BioMed Central Ltd.

This is an Open Access article distributed under the terms of the Creative Commons Attribution License (http://creativecommons.org/licenses/by/2.0), which permits unrestricted use, distribution, and reproduction in any medium, provided the original work is properly cited.

\begin{abstract}
Background: The contribution of individual organs to the whole-body adaptive response to fasting has not been established. Hence, gene-expression profiling, pathway, network and gene-set enrichment analysis and immunohistochemistry were carried out on mouse liver after 0, 12, 24 and 72 hours of fasting.

Results: Liver wet weight had declined $\sim 44, \sim 5, \sim 11$ and $\sim 10 \%$ per day after 12, 24, 48 and 72 hours of fasting, respectively. Liver structure and metabolic zonation were preserved. Supervised hierarchical clustering showed separation between the fed, 12-24 h-fasted and $72 \mathrm{~h}$-fasted conditions. Expression profiling and pathway analysis revealed that genes involved in amino-acid, lipid, carbohydrate and energy metabolism responded most significantly to fasting, that the response peaked at 24 hours, and had largely abated by 72 hours. The strong induction of the urea cycle, in combination with increased expression of enzymes of the tricarboxylic-acid cycle and oxidative phosphorylation, indicated a strong stimulation of amino-acid oxidation peaking at 24 hours. At this time point, fatty-acid oxidation and ketone-body formation were also induced. The induction of genes involved in the unfolded-protein response underscored the cell stress due to enhanced energy metabolism. The continuous high expression of enzymes of the urea cycle, malate-aspartate shuttle, and the gluconeogenic enzyme Pepck and the re-appearance of glycogen in the pericentral hepatocytes indicate that amino-acid oxidation yields to glucose and glycogen synthesis during prolonged fasting.
\end{abstract}

Conclusion: The changes in liver gene expression during fasting indicate that, in the mouse, energy production predominates during early fasting and that glucose production and glycogen synthesis become predominant during prolonged fasting. 


\section{Background}

Abstinence or absence of food requires the body to recruit metabolites from pre-existing stores. Based on the rate of weight loss, nitrogen excretion, concentration of plasma metabolites and resting metabolic rate, the body is thought to pass through three successive adaptive phases during fasting [1] that have been associated with the primary fuel that is putatively available to the tissues (e.g [2$5])$. During the brief postabsorptive period, the rate of weight loss is relatively high ( $24 \%$ per day in mice [6], $\sim 10 \%$ per day in rats $[7,8]$, and $\sim 2 \%$ in humans [9]). The decreasing insulin levels induce glycogenolysis (primarily muscle and liver) and lipolysis $[10,11]$ to support circulating glucose, triglyceride and cholesterol levels [8]. During the subsequent "coping" phase, the loss of body mass is slower ( $~ 7 \%$ per day in mice, $\sim 6 \%$ per day in rats [7], and $\sim 1 \%$ in humans [12]). This state, which can be maintained for several weeks in humans $[13,14]$, for almost a week in rats [7], and for 2-3 days in mice [6], is thought to depend, at least in humans, on lipids as the main fuel source. However, amino-acid oxidation and, hence, protein catabolism remains necessary for continuous anaplerosis of the TCA cycle [14]. It is widely accepted that muscle is a main source of amino acids from protein catabolism, that protein catabolism is maintained by an increased in the circulating levels of glucocorticosteroids, and that glutamine and alanine are the main carriers of this energy source $[15,16]$ to the intestine, liver and kidney $[2,5,7,17-19]$. As a result, total splanchnic glucose production amounts to approximately 80 grams daily in humans after several weeks of starvation [13]. Despite this enhanced glucose production, but reflecting the enhanced fatty-acid oxidation and ketone-body synthesis in muscle and splanchnic region $[5,20]$, the brain gradually switches to ketone-body oxidation after several weeks of starvation $[21,13]$. During the preterminal phase, finally, the rate of loss of body weight may increase again ( $~ 9 \%$ in rats [7]). Because the fat stores are depleted, proteolysis remains the sole, nonsustainable source of fuel.

The maintenance of the fuel supply during fasting requires an extensive exchange of metabolites from organs that break down the stores of fats or proteins to organs that consume these metabolites. This exchange mainly occurs as glucose, lactate, amino acids, triglycerides and ketone bodies. The question that arises from these global findings concerns the contribution of individual organs to the whole-body adaptive response to fasting. Our previous study of the effects of fasting on the small intestine [6] suggested, in comparison with that of liver [22] and muscle $[23,24]$, an organ-specific response to fasting. Our study [6] included both shorter and longer periods of fasting than earlier published studies [22-24]. The aim of the current study was, therefore, to determine the characteristics of gene-expression profile of mouse liver between 0 and 72 hours of fasting, using a genome-wide transcriptomics approach. Our findings show that the adaptive response of the liver peaks around 24 hours after food withdrawal and, unexpectedly, declines thereafter. The major components of the response were fatty-acid $\beta$-oxidation and ketone-body synthesis, and oxidative and energy metabolism during the first 24 hours of fasting, and glycogen synthesis and the urea cycle throughout the entire fasting period.

\section{Results}

\section{Effects of food withdrawal on liver structure}

During the first 12 hours of fasting, mice lost 12\% of their body weight (that is, $24 \%$ if expressed on a per-day basis). Thereafter, weight loss remained steady at a rate of $\sim 7 \%$ per day, so that mice had lost $\sim 30 \%$ of their initial weight after 72 hours of fasting (Figure 1A). Note that we expressed daily differences in the rate of weight loss on a per-day basis to define a common denominator for the 12 h- and 24 h-fasted animals. Liver wet weight declined more than body weight (Figure 1A), especially during the first 12 hours of fasting, and amounted to $\sim 44, \sim 5, \sim 11$ and $~ 10 \%$ per day after 12, 24, 48 and 72 hours of fasting, respectively. After 72 hours, the liver had, therefore, lost almost $50 \%$ of its initial weight. The basic architecture of the liver lobules (Figure 1B, HA) and the zonation of gene expression as studied by the expression of glutamine synthetase and carbamoylphosphate synthetase (Figure 1B, GS and CPS) remained unaffected. Staining for the appearance of active-caspase 3 revealed no changes in the number of apoptotic cells upon fasting, not even after 72 hours (data not shown). In agreement, the apoptotic genes that were represented on the microarrays showed no significant change in expression in fasted compared to fed mice. Since there was no reduction in the number of liver cells during fasting, we took two approaches to estimate the decrease in average cell size. The summation of 50 hepatocyte diameters in three 72 hours fasted and three control animals, amounted to $25 \%$ reduction in cell diameter. Based on the liver wet weight, the average cell diameter decreased $20 \%$ in the fasted animals.

\section{Effects of fasting on metabolism}

Ammonia levels had increased 2.0-, 3.7- and 5.2-fold after 24,48 and 72 hours of fasting, respectively $(\mathrm{P}<0.005$; Figure $2 \mathrm{~A})$. Glucose and lactate concentrations remained stable until $48 \mathrm{~h}$ of fasting, but decreased 34 and $43 \%$, respectively $(\mathrm{P}<0.05$ and 0.005 , respectively, Figure $2 \mathrm{~A})$ in the next 24 hours. The plasma concentration of many amino acids changed at some time point of fasting, but only the changes in the concentration of taurine showed a trend with time (Figure 2B and Supplementary Table 1, Additional file 1). Accumulation of taurine helps protect cells from hypertonicity [25], as may occur during shrinkage of fasting hepatocytes. 


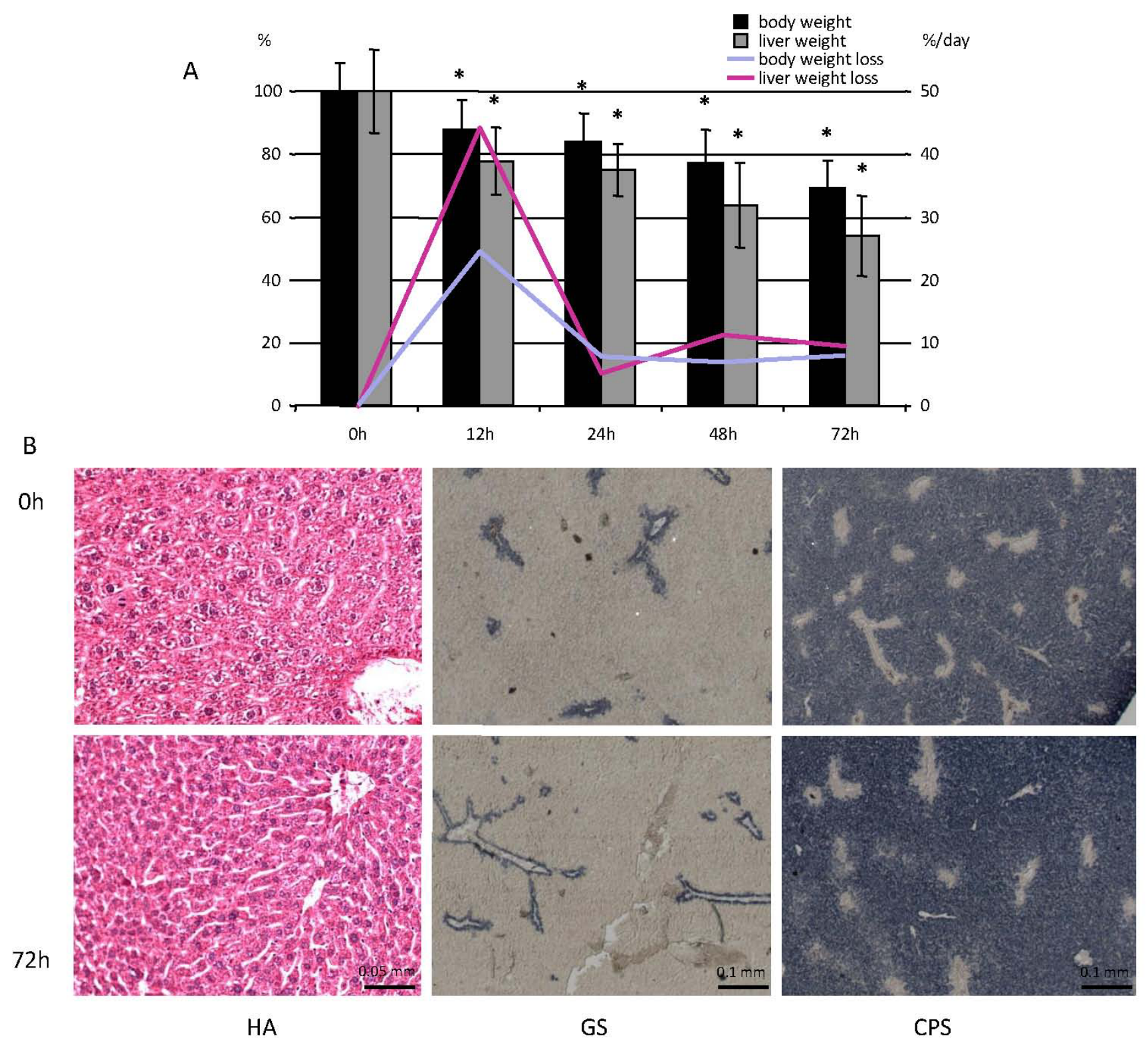

Figure I

Macro- and microscopic analysis of the fasting liver. A) Change in whole-body and liver weight during fasting as percentage of fed weight $(n \geq 8)$. Asterisks label significant changes $(P<0.01)$. The blue and pink lines represent the daily percentual change in body and liver weight, respectively, with the percent weight loss per day shown on the secondary $y$-axis. B) Histology of fed and 72 hour-starved livers (upper and lower panel, respectively). The sections were stained with hematoxylin and azophloxin (HA), and for the presence of glutamine synthetase (GS; pericentral expression) and carbamoylphosphate synthetase (CPS; periportal expression). The figures show that lobular architecture and metabolic zonation are unaffected by fasting. Bars: $0.1 \mathrm{~mm}$.

\section{Global gene-expression profile in the liver}

To gain a comprehensive overview of the physiological response of the liver to fasting, whole-genome measurements were made. Compared to the fed group, 201, 504 and 119 transcripts, including expressed sequence tags and RIKEN sequences, met our boundary condition for significance ( $\geq 1.4$-fold change with $\mathrm{P}<0.01$ ) after 12,24 , and 72 hours of fasting, respectively (Figure 3A; for a complete list of more than 1.4-fold up- or downregulated genes, see Additional file 2). The dendrogram generated by supervised hierarchical clustering (Figure 3B) shows a clear separation between fed and fasted conditions. 


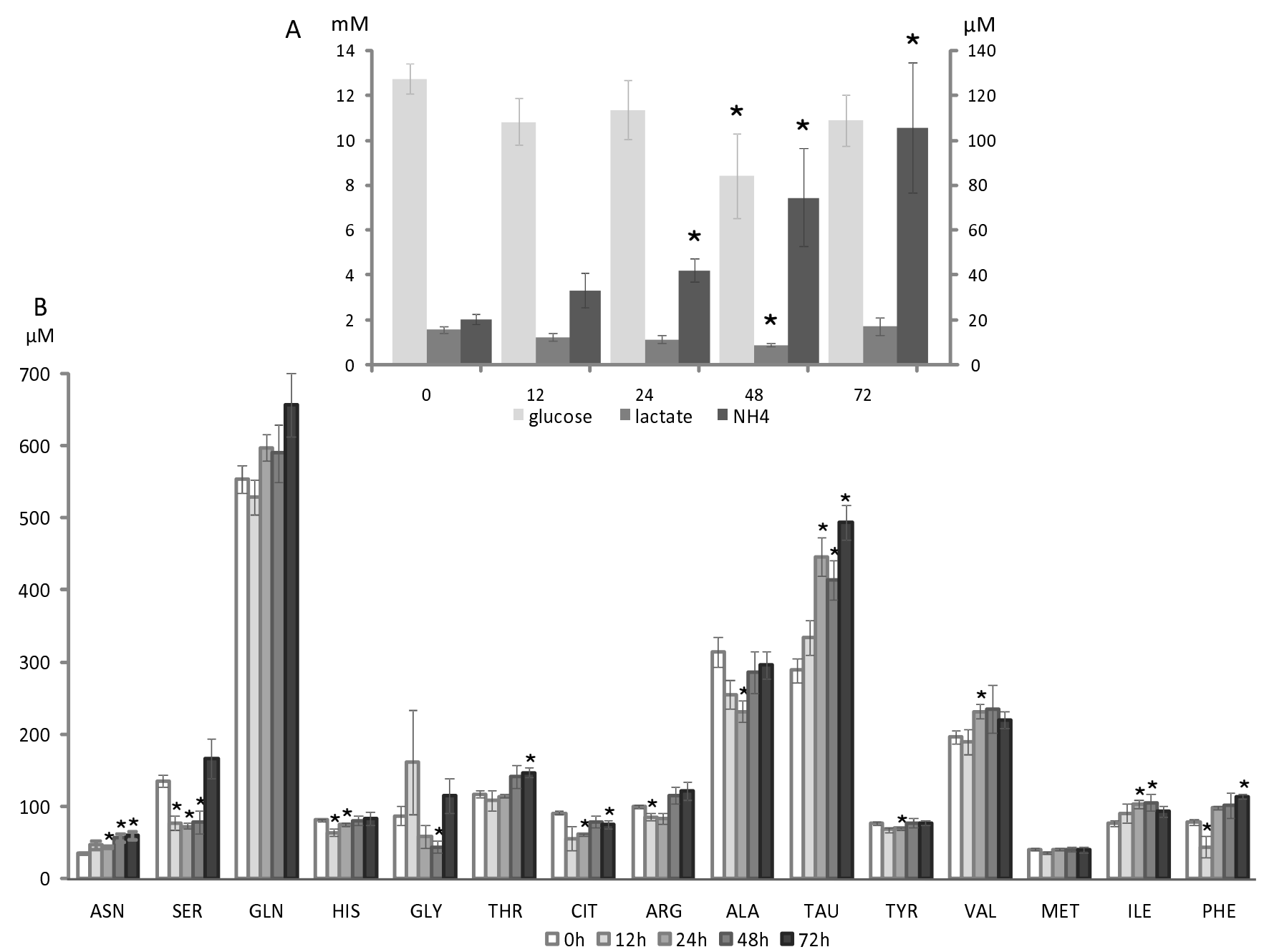

Figure 2

Changes in plasma metabolite concentrations during fasting. A) Glucose and lactate (mM, primary $\mathrm{Y}$-axis), and ammonia concentrations ( $\mu \mathrm{M}$, secondary $Y$-axis) after $0,12,24,48$ and 72 hours of fasting. $B$ ) Adaptive changes in concentrations of a selection of amino acids during fasting (those without significant change between any two time points were left out). For all the metabolites measured: $8<\mathrm{n}<12$; bars represent SEM and the asterisks identify significant changes $(P<0.05)$ in comparison to the fed condition.

Among the arrays coming from fasted animals, those from 72 hours stand out, while the branches of the two earlier time points are intertwined, indicating that expression profiles are rather similar after 12 and 24 hours of fasting. This is also reflected in the Venn diagrams where the overlap between 12 and 24 hours is larger than the overlap with 72 hours.

\section{Global analysis reveals a strong early and an abated late response to fasting}

We used GenMAPP and, in particular, MetaCore ${ }^{\mathrm{TM}}$ software to deduce the biological processes that change with an increasing duration of fasting from the liver transcriptome data. In MetaCore, the degree of association of the uploaded datasets with predefined metabolic pathways is defined by P-values, with lower P-values being more relevant. The expression of 465 genes that met our thresholds (56\%) could be linked to the MetaCore ${ }^{\mathrm{TM}}$ suite. Their distribution across time points is shown in Figure 4A. The graphs show the numbers of unique, similar and common genes for all three, and for two initial time points separately, showing that the response to fasting at 24 hours was similar to, but more pronounced than that at 12 hours.

We performed gene-set enrichment analysis in three different functional ontologies using MetaCore ${ }^{\mathrm{TM}}$ : cellular processes, biological processes and canonical pathways. 
Table I: Top I0 canonical pathways influenced by fasting

\begin{tabular}{|c|c|c|c|}
\hline pathway & pathway group & p- value & genes \\
\hline glycolysis and gluconeogenesis & metabolic maps/carbohydrate metabolism & $2.32 \mathrm{e}-07$ & $9 / 36$ \\
\hline urea cycle & metabolic maps/amino-acid metabolism & $2.6 \mathrm{le}-07$ & $8 / 27$ \\
\hline PPAR $\alpha$ regulation of lipid metabolism & regulation of metabolism/regulation of lipid metabolism & $3.57 e-07$ & $8 / 28$ \\
\hline peroxisomal straight-chain fatty-acid $\beta$-oxidation & metabolic maps/lipid metabolism & $2.5 \mathrm{le}-06$ & $5 / 10$ \\
\hline mitochondrial long-chain fatty-acid $\beta$-oxidation & metabolic maps/lipid metabolism & $2.74 \mathrm{e}-06$ & $6 / 17$ \\
\hline metabolism of sulphur-containing amino acids & metabolic maps/amino-acid metabolism & $4.02 \mathrm{e}-06$ & $6 / 18$ \\
\hline peroxisomal branched-chain fatty- acid oxidation & metabolic maps/lipid metabolism & $8.04 \mathrm{e}-06$ & $6 / 20$ \\
\hline taurine metabolism & metabolic maps/amino-acid metabolism & $1.96 \mathrm{e}-05$ & $6 / 23$ \\
\hline mitochondrial unsaturated fatty- acid $\beta$-oxidation & metabolic maps/lipid metabolism & $2.69 e-05$ & $5 / 15$ \\
\hline TCA & metabolic maps/amino-acid metabolism & $1.25 \mathrm{e}-04$ & $4 / 20$ \\
\hline
\end{tabular}

Canonical pathways represent a set of about 500 signalling and metabolic maps in the MetaCore suit. All maps are drawn from scratch by GeneGo annotators and manually curated.

Based on the Gene Ontology categorization of cellular processes, fasting predominantly affected the metabolic processes, in particular the carboxylic-acid metabolizing processes, lipid and glucose metabolism. The enrichment analysis for biological processes showed, more specifically, that genes involved in amino-acid, lipid, carbohydrate and energy metabolism responded most significantly to fasting (Figure $4 \mathrm{~B}$ ). The graph presents Pvalues as parameter of the likelihood that coordinate changes in the pathways shown were indeed present at the different time points of fasting. As statistical parameter, the P-value encompasses no variation. The changes in all processes except amino-acid metabolism showed a response that peaked at 24 hours after food withdrawal and declined thereafter. The response during the late phase of fasting was dominated by amino-acid metabolism, although lipid and carbohydrate metabolism remained significantly regulated. The Figure further reveals that the changes in energy metabolism were significant at 24 hours of fasting only. The common denominator of the overall fasting response was, therefore, metabolism of amino acids, carbohydrates, and lipids.

\section{Regulated pathways}

Since the global analysis does not reveal a direction in the changes and lacks functional detail, we scrutinized the pathways with most pronounced regulation for functional implications. A list of the 10 top-scoring canonical pathways, shown in Table 1, points to gluconeogenesis, urea synthesis, and PPAR $\alpha$-regulated fatty-acid oxidation as the major characteristics in the response of the liver to fasting.

\section{Amino-acid catabolism and urea synthesis}

Of all the pathways studied in the liver, the adaptive changes in amino-acid metabolism persisted throughout the fasting period (Figure 4B). Of the enzymes in this group, those of the urea cycle were upregulated at all three time points (Figure 5). Among the genes consistently affected were argininosuccinate synthetase 1 (Ass1, Assy; 3.7-, 2.5- and 4.5-fold upregulated) and argininosuccinate lyase (Asl, Arly; 5.0-, 5.8-, and 12-fold upregulated at 12, 24 and 72 hours, respectively. The first and rate-determining enzyme of urea cycle, carbamoylphosphate synthetase (Cps), was not represented on the microarrays, but its expression level, as estimated by qPCR, was increased 3.5fold at all 3 time points (manually added to Figure 5). Urea synthesis occurs in periportal hepatocytes, whereas ammonia detoxification via glutamine synthesis occurs pericentrally. Genes for the pericentral enzymes ornithine-aminotransferase (Oat) and proline dehydrogenase (Prodh), which provide glutamate for glutamine synthesis, were upregulated 2.5-, 3.0- and 3.0-fold and 2.1-, 2.5- and 2.0- fold at 12, 24 and 72 hours, respectively. Glutamine synthetase (Glns) itself was, however, not regulated.

Remarkably, the expression of amino-acid catabolizing enzymes themselves was barely affected by fasting. Only the degradation of branched-chain keto-acids (products of branched-chain amino-acid transamination elsewhere) was upregulated, as shown by the upregulation of acetylcoenzyme A dehydrogenase, medium chain (Acaddm), enoyl-coenzyme A, hydratase/3-hydroxyacyl-coenzyme (Ehhadh), hydroxyacyl-coenzyme A dehydrogenase, short chain (Hadhsc), acetyl-coenzyme A acyltransferase 1 (Acaa1), and 3-hydroxy-3-methylglutaryl-coenzyme A lyase $(\mathrm{Hmgcl})$ - all within first the 24 hours (Figure 5). This finding suggests that the adaptations in amino-acid catabolism during fasting mainly occur outside the liver. Since neither glutamate-pyruvate transaminase nor ammonia-inducible liver glutaminase were upregulated, the capacity of the liver to deaminate the amino-carriers alanine and glutamine must have been sufficient.

\section{TCA cycle and electron-transport chain}

The strong induction of the urea cycle suggests a strong stimulation of amino-acid oxidation or gluconeogenesis. In agreement with this hypothesis, both the expression of 

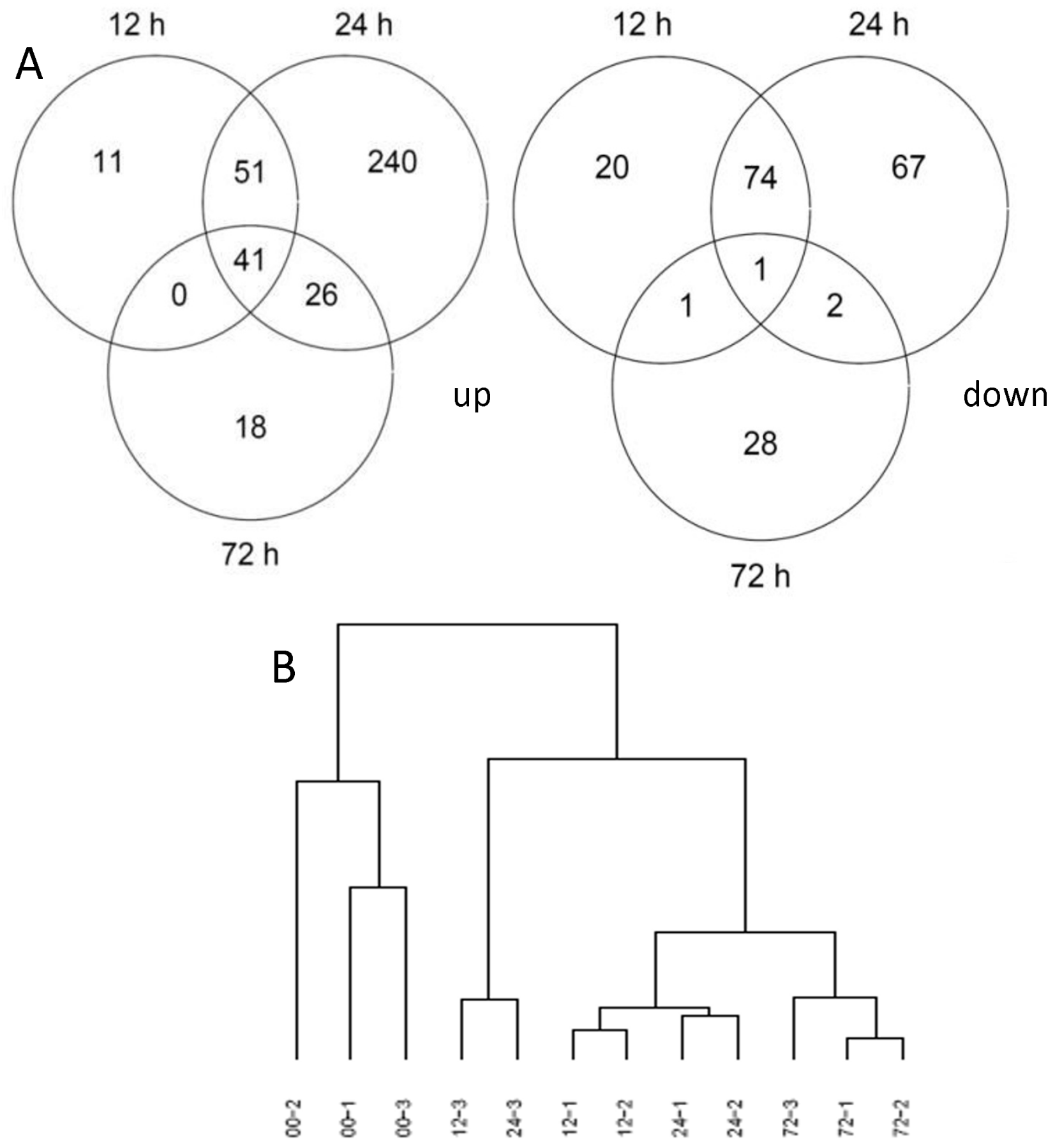

Figure 3

Number of genes in the liver that are affected by fasting. A) Number of differentially expressed genes ( $\geq$ I.4-fold change in expression; $P<0.01$ ) at each time point studied. The left-sided Venn diagram shows the number of up-regulated and the right-sided diagram the number of down-regulated genes. Genes that were changed in expression at more than one time point are shown in the overlapping areas. B) Supervised hierarchical cluster based on correlation and complete linkage. 
A

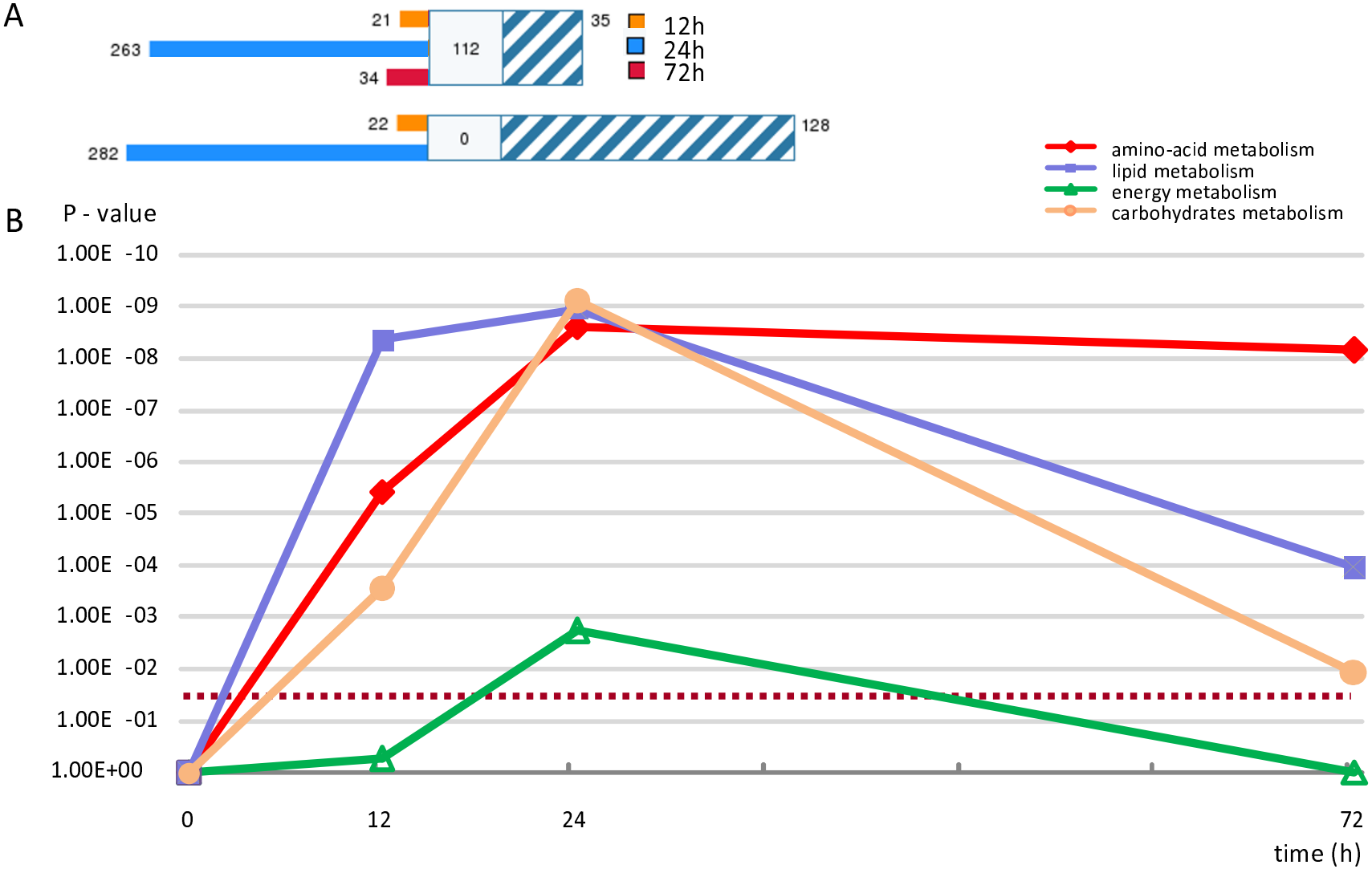

Figure 4

Adaptive changes in metabolic processes in the liver during fasting as analyzed by MetaCore ${ }^{\mathrm{TM}}$ software. A) The gene content imported to MetaCore ${ }^{\mathrm{TM}}$ is aligned between the time points. The parameters for comparison are $\geq \mathrm{I} .4$ fold change and $\mathrm{P}<0.0 \mathrm{I}$, and the annotation allowed for $56 \%$ of such genes to be linked. The unique genes changed at each of the time points are marked as colored bars (orange, blue and red for 12, 24 and 72 hours, respectively). The set of common genes, changed in all three conditions, is shown in blue-white hatching. The middle white box represents the similar genes (present in 2 out of 3 data points). The upper panel represents differentially regulated genes in all three time points of fasting, while the lower one shows uniquely and commonly differentially expressed genes for 12 and 24 hours of fasting. B) Four groups of metabolic processes changed significantly in response to fasting, with the response of all peaking at 24 hours. The $Y$-axis shows the significance of change, while the $X$-axis represents duration of fasting. The $P$-values of the pathways are calculated using the hypergeometric distribution, where the $\mathrm{P}$-value represents the probability of a particular mapping arising by chance, given the numbers of genes in the set of all genes in pathways, genes in a particular pathway, and genes in the present experiment. The pathways are grouped into processes as defined in MetaCore ${ }^{\mathrm{TM}}$ (version 4.3, build 9787). The dotted line represents the 0.05 significance threshold.

enzymes of the tricarboxylic-acid (TCA) cycle and oxidative phosphorylation were induced in fasted liver, again mainly at 24 hours. Aconitase 2 (Aco2), isocitrate dehydrogenase $3 \beta\left(\mathrm{NAD}^{+}\right)(I d h 3 b)$, oxoglutarate dehydrogenase $(O g d h)$, dihydrolipoamide S-succinyltransferase (Dlst), fumarate hydratase 1 (Fh1) and malate dehydrogenase 1 (Mdh1) were all upregulated at 24 hours of fasting (1.9-, 1.5-, 3.1-, 2.0-, 1.4- and 1.6-fold, respectively; Figures 5 and 6), indicating an increased capacity of the cycle. Dlst and Fh1 were 2.0 and 1.6 times induced at 12 hours of fasting, while Aco 2 expression was also 1.8-fold increased at 72 hours.
In agreement with an increased capacity of the TCA cycle, the expression of the genes of the electron-transport chain was strongly stimulated (Figure 7; a legend for the MetaCore canonical pathways is provided in Additional file 3). Four genes belonging to NADH-ubiquinone oxidoreductase complex: NADH dehydrogenase [ubiquinone] $1 \alpha$ subcomplex subunit 10 (Ndufa10), NADH dehydrogenase [ubiquinone] $1 \alpha$ subcomplex subunit 13 (Ndufa13), $\mathrm{NADH}$ dehydrogenase [ubiquinone] flavoprotein 1 (Ndufv1) and NADH dehydrogenase [ubiquinone] flavoprotein 2 (Ndufv2), were all approximately 1.6-fold upregulated. Expression of the genes of the ATP synthase 


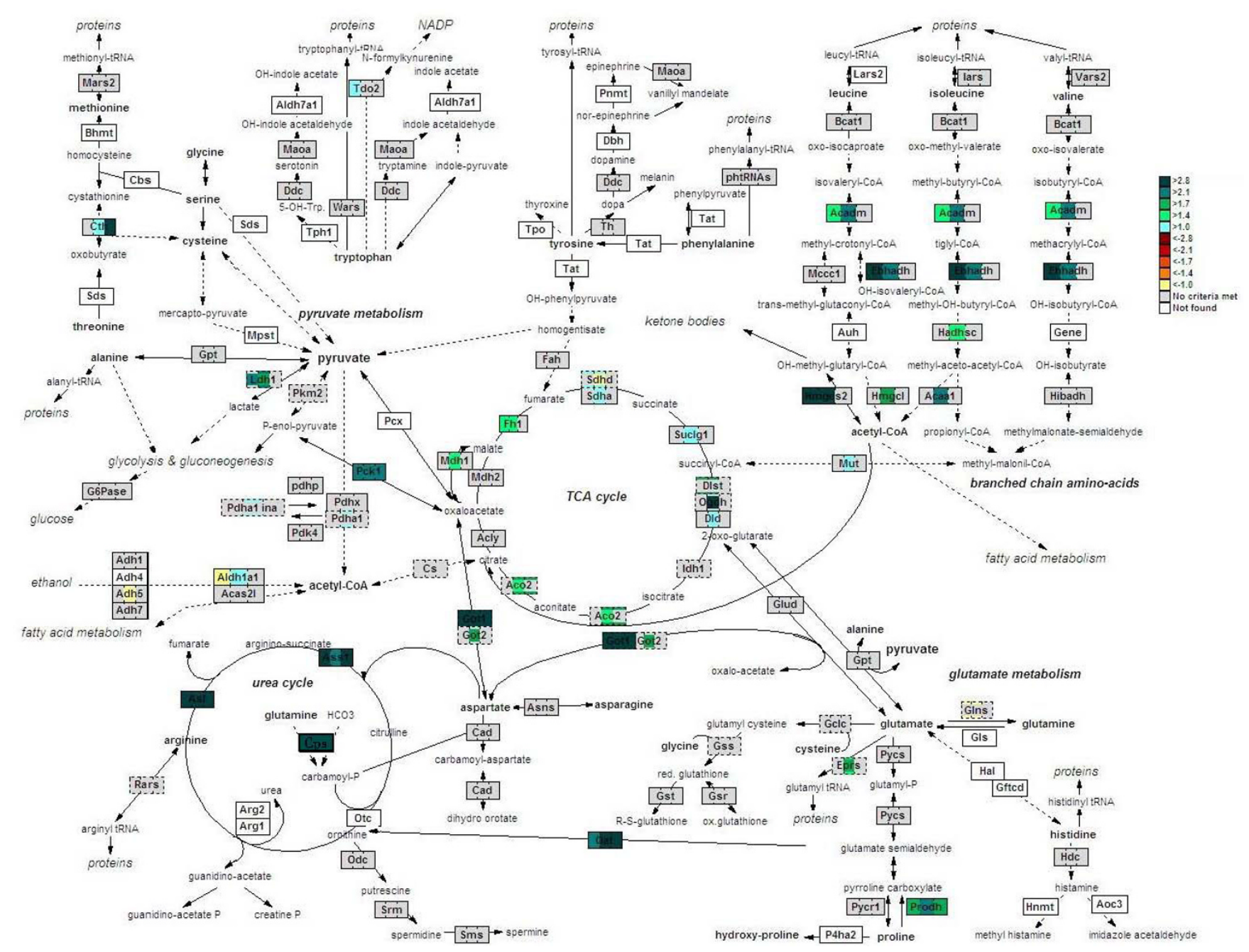

\section{Figure 5}

Amino-acid catabolism in fasting liver. The map was created in the GenMAPP suite to show a comprehensive overview of amino-acid metabolism in response to fasting. Warm colors (from yellow to red) represent down-regulation, while cold colors (light blue to dark green) indicate an induction (see scale on the right border of the figure). Gray indicates no significant change. Genes not coupled to reporters on the array are shown in white. Genes represented by more than one sequence on the array are shown in dash-lined boxes, with the level of change depicted by the colored line surrounding the field. Each genebox is split into 3 units, representing (from left to right) a change in expression after 12,24 and 72 hours of fasting compared to fed animals.

complex, ATP synthase subunit $\alpha$ (Atp5a1), ATP synthase $\delta$ chain $(A t p 5 d)$ and ATP synthase lipid-binding protein (Atp5g1), was 1.6-1.9-fold induced at 24 hours. Ubiquinol-cytochrome-c reductase complex core protein 1 (Uqcrc1) was 2.2-fold upregulated after 24 hours, whereas the energy-dissipating uncoupling protein 2 (Ucp2) was 1.8-fold downregulated at this time point (Figure 8). Taken together, these data indicate that the capacity for ATP synthesis in the liver is strongly upregulated during the first day of food deprivation.

\section{Gluconeogenesis}

Phosphoenolpyruvate carboxykinase 1 (Pepck1), a key enzyme in the gluconeogenic route, was upregulated 2.0, 2.5- and 2.7-fold on the microarrays and 3.2-, 3.2, and 2.9-fold in the qPCR measurements at 12,24 and 72 hours of fasting, respectively (Figures 5 , and 6 , and Table $2)$. Cytosolic glutamate oxaloacetate transaminase 1 (Got1) was also strongly upregulated at all three time points (5-, 6-, and 21-fold). In addition, malate dehydrogenase $(M d h)$ and mitochondrial glutamate oxaloacetate transaminase (Got2) were induced (1.6- and 1.8-fold 


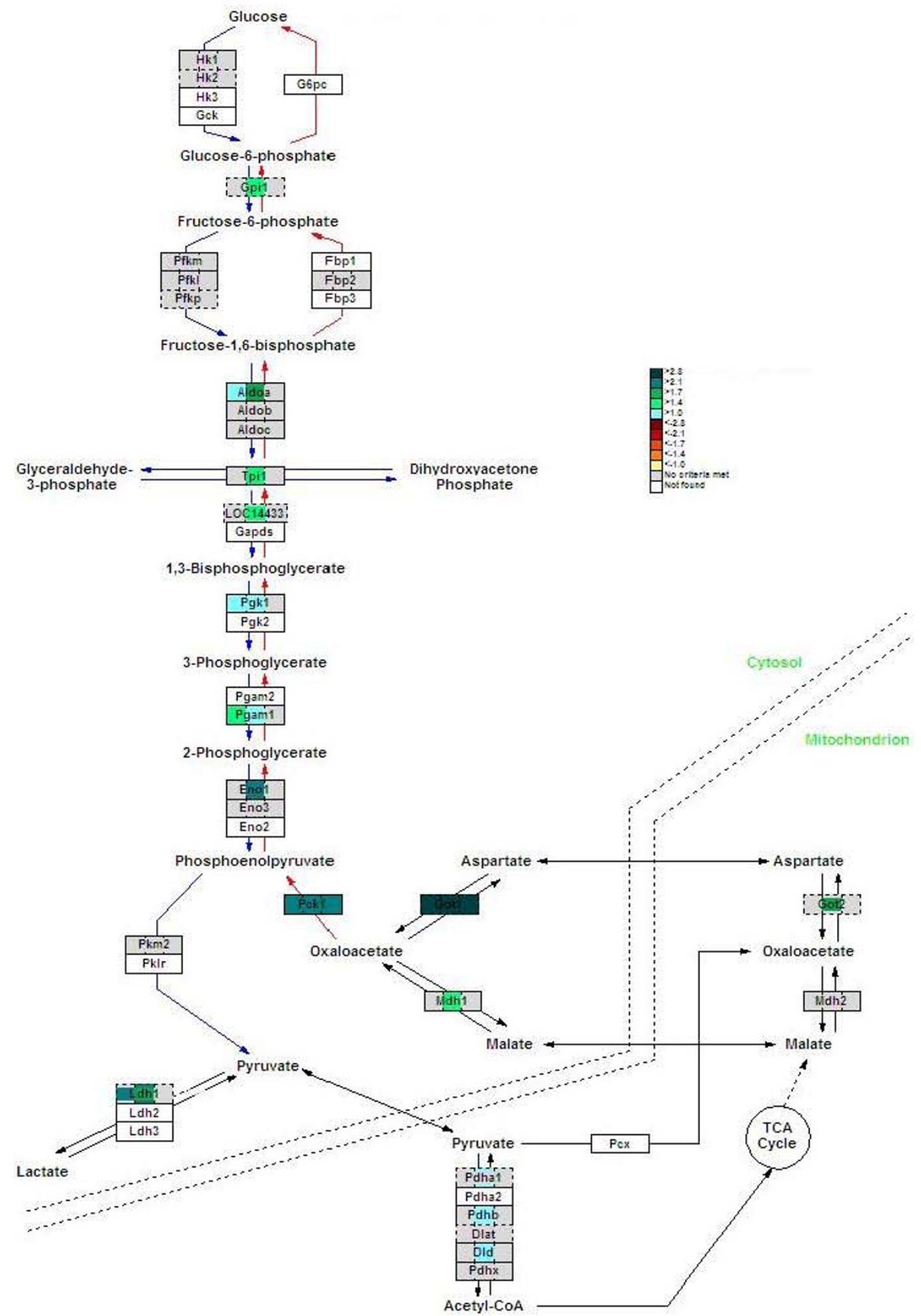

Figure 6

Fasting upregulates genes of the malate-aspartate shuttle and the gluconeogenic enzyme Pepckl in mouse liver. The color code of the GenMAPP view is the same as in Figure 5. 


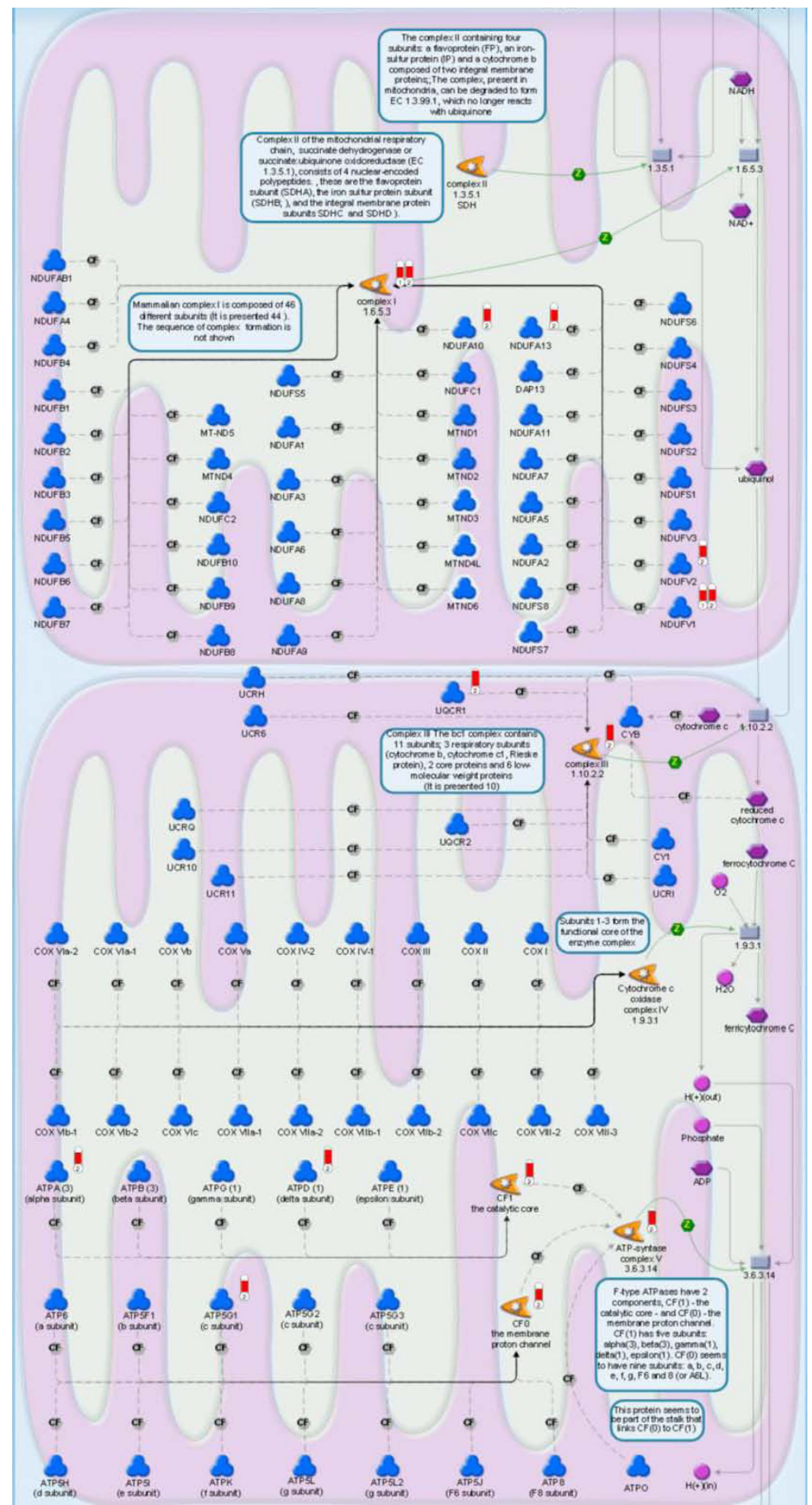

Figure 7

Electron-transport chain. Experimental data are visualized on a MetaCore map as blue (for downregulation) and red (upregulation) histograms ('thermometers'). The height of the histogram corresponds to the relative expression value for a particular gene, with numbers I, 2 and 3 representing 12, 24 and 72 hours of fasting, respectively. A legend for the MetaCore $^{\mathrm{TM}}$ canonical pathways is provided in Additional file 3. 


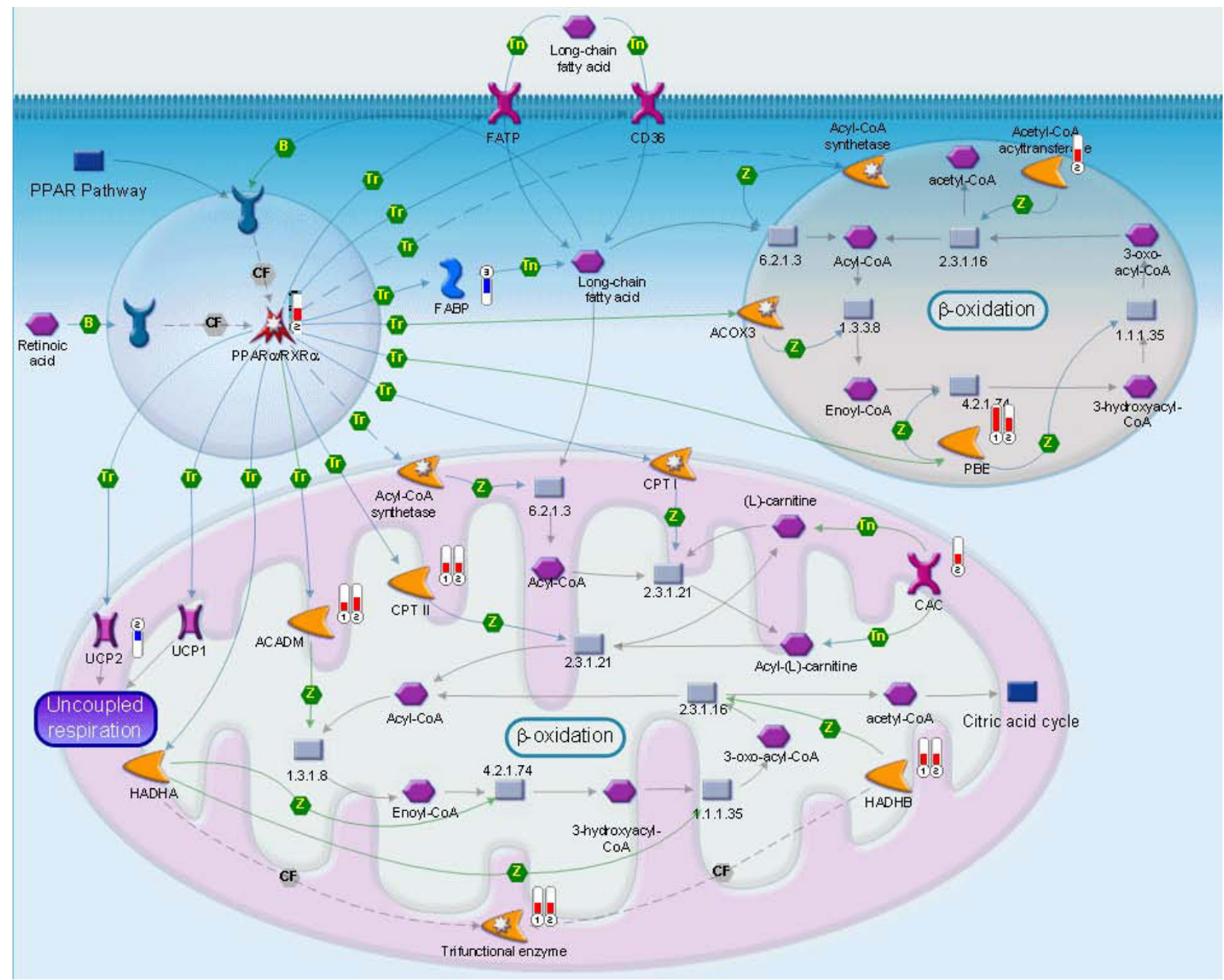

Figure 8

PPAR $\alpha$ regulation of lipid metabolism in fasting. The figure description is the same as in Figure 7.

respectively). Together, these data suggest an increased capacity of the malate-aspartate shuttle across the mitochondrial membrane, which would accommodate an enhanced carbon flux from the mitochondria.

All other steps that were affected by fasting were shared by the glycolytic and gluconeogenic pathways and were reg-

Table 2: Comparison of intestinal and liver Pepckl expression in fasting by $q R T P C R$, expressed in relative units after normalization by $18 \mathrm{~S}$ expression $(n=6)$

\begin{tabular}{cccc}
\hline time (h) & small intestine & liver & ratio \\
\hline $\mathbf{0}$ & 18 & 85 & 0.21 \\
$\mathbf{1 2}$ & 15 & 278 & 0.05 \\
$\mathbf{2 4}$ & 51 & 277 & 0.18 \\
$\mathbf{7 2}$ & 128 & 247 & 0.52
\end{tabular}

ulated during the first day of fasting only (Figure 6). Phosphoglycerate mutase 1 (Pgam1) was 1.4-fold upregulated at 12 hours of fasting, while glucosephosphate isomerase 1 (Gpi1), aldolase 1A isoform (Aldoa), triosephosphate isomerase 1 (Tpi1), glyceraldehyde-3-phosphate dehydrogenase (Gapdh) and enolase $1 \alpha$ (Eno1) were 1.5-, 1.7-, 1.5, 1.7-, 2.1- fold upregulated at 24 hours of fasting, respectively. These data indicate that the enhanced capacity of the gluconeogenic pathway would largely depend on enhanced TCA and malate-aspartate cycling, and that this adaptive response in gene expression might be restricted to a single day in the mouse.

\section{Liver glycogen accumulation upon prolonged fasting}

The near total return to "normalcy" of gene expression at 72 hours (only the genes for urea cycle enzymes, gluta- 
mate-synthesizing enzymes, and Pepck1 remained induced) was striking. Because glucose-6-phosphatase expression was not upregulated, we explored the possibility that glucose precursors were channelled into glycogen. As expected, (amylase-sensitive) periodic acid-Schiff (PAS) staining showed the complete disappearance of glycogen from the liver after 12 hours of fasting (Figure 9), but some staining had returned at 24 hours and intense staining was seen in 72-hours fasted liver. Whereas glycogen was localized around the portal veins in fed liver, it was deposited exclusively around the central veins after 72 hours of fasting, with sharp borders towards the empty cells.

\section{Fatty-acid catabolism and ketone-body synthesis}

The enhanced expression of fatty-acid catabolizing enzymes was also limited to the initial phase of fasting. The expression of the transcription factor Ppar $\alpha$, a major regulator of fatty-acid oxidation, was 2.1-fold upregulated at 24 hours of fasting (Figure 8). Furthermore, the mitochondrial carnitine/acylcarnitine fatty-acid translocase (Cac or Slc25a20) was 1.6-fold upregulated at 12 hours, while carnitine palmitoyltransferase 2 (Cpt2) on the inner mitochondrial membrane was 1.8 -fold upregulated at both 12 and 24 hours of fasting. However, the expression of Cpt1, which is present on the outer mitochondrial membrane and is sensitive to malonyl-CoA inhibition, remained unchanged. The 4 acyl-coenzyme A dehydrogenases (Acad $-v,-l,-m$ and $-s h$ ), involved in oxidation of very long-, long-, medium- and short-chain fatty acids, were all upregulated in the first 24 hours of fasting (1.5-2.6 fold). The $\beta$-subunit of the trifunctional protein (Hadhb) was 2and 2.1-fold upregulated at 12 and 24 hours, respectively, while another subunit, hydroxyacyl-coenzyme A dehydrogenase, short chain (Hadhsc) showed increased expression after 24 hours of fasting only, indicating altogether a strong stimulation of fatty-acid oxidation at the geneexpression level during the first day of fasting.
The expression of HMGCoA synthase 2 (Hmgcs2) was also strongly stimulated during the first day of fasting (3.4and 2.9-fold at 12 and 24 hours, respectively; Figure 5), indicating an increased capacity of the synthesis of ketone bodies from acetyl-CoA. This process is further facilitated by increased expression of genes involved in branchedchain keto-acid degradation (Acadm, Hadhsc and Ehhadh; see section on amino-acid catabolism) at 12 and 24 hours. Interestingly, neither the cytoplasmic HMGCoA synthase (Hmgcs1) nor HMGCoA reductase (Hmgcr), the key enzyme in de novo cholesterol synthesis pathway [26], have changed the expression levels in fasted liver.

Among the genes involved in fatty-acid synthesis, enoyl coenzyme A hydratase domain containing $3(E c h d c 3)$ was 1.6 and 1.7 -fold downregulated at 12 and 24 hours, while stearoyl-coenzyme A desaturase 1 ( $S c d 1)$ showed a 2.6fold decrease in expression at 72 hours of fasting. These data underscore the importance of enhanced lipid catabolism in the liver, which, in the mouse, apparently occurs during the first day of fasting only.

\section{Oxidative stress and unfolded protein response}

The enhanced expression of TCA cycle and oxidativephosphorylation enzymes often causes oxidative stress. Indeed, cytosolic superoxide dismutase (Sod1) was 2.2fold upregulated after 24 hours, and the early growth response protein 1 (Egr1), its transcriptional regulator [27], 2.9-fold. Furthermore, catalase (Cat) and stress-regulated mitogen-activated protein kinase 14 (Mapk14) were both 1.4-fold upregulated at this time point. In addition, metallothionein 1 gene, known to be involved in protection against oxidative stress and metal toxicity [28], was intensely upregulated (8.6-, 5.5- and 13.5 -fold, at 12, 24 and 72 hours, respectively).

Interestingly, the 3 top-scoring processes obtained from a biological-process enrichment analysis all belonged to the
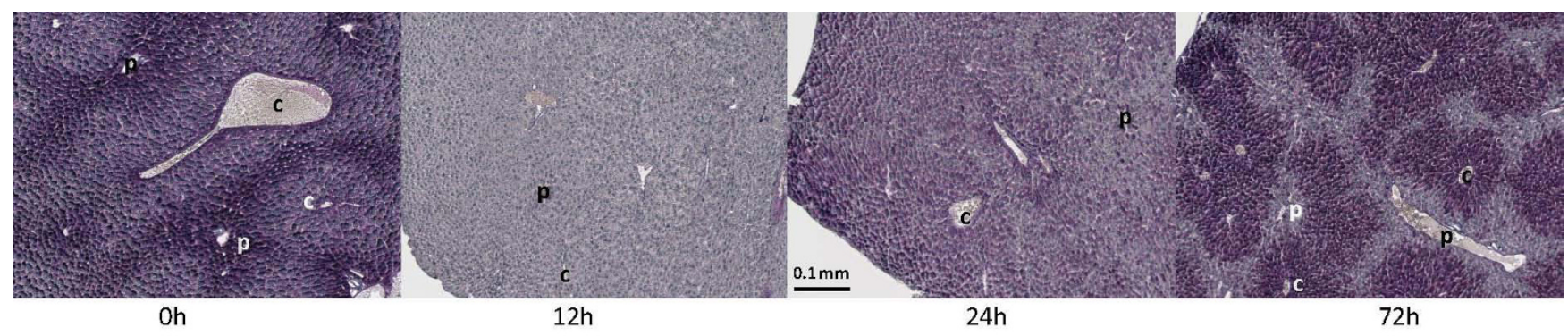

Figure 9

Glycogen storage in mouse liver during fasting. In fed liver, periportal hepatocytes contain most glycogen (left panel). Twelve hours of fasting totally depletes the glycogen stores, but at 24 hours, glycogen starts to re-accumulate and has accumulated to high levels in pericentral hepatocytes after 72 hours of fasting (right panel). Portal and central veins are depicted by letters "p" and "c", respectively. Bar: $0.1 \mathrm{~mm}$. 
unfolded-protein response (endoplasmic reticulum (ER) stress). To present the relevant data in a single figure, we created a network using the shortest-path algorithm (Figure 10). The resulting network provides links based on the known interaction data between the nodes from the query data set, and also between the nodes that regulate the given genes or metabolites. It shows 8 heat-shock and 6 other proteins, all upregulated 1.5-2.5 fold, indicating upregulation of this stress-response pathway in fasted liver. Downstream of the ER stress pathway, proteasome degradation was also upregulated, but again only after 24 hours of fasting (Figure 11). A list of these and some additional genes regulated in the ER stress and proteasome degradation, with their change level, is shown in Table 3.

\section{Discussion}

The major architectural feature of fasting liver is a pronounced decline in cell size (down to $\sim 75 \%$ of its fed diameter after 3 days of fasting) rather than a loss of cell number. In addition, the liver's metabolic zonation in upstream, periportal and downstream, pericentral regions remains intact. These findings indicate that the liver can

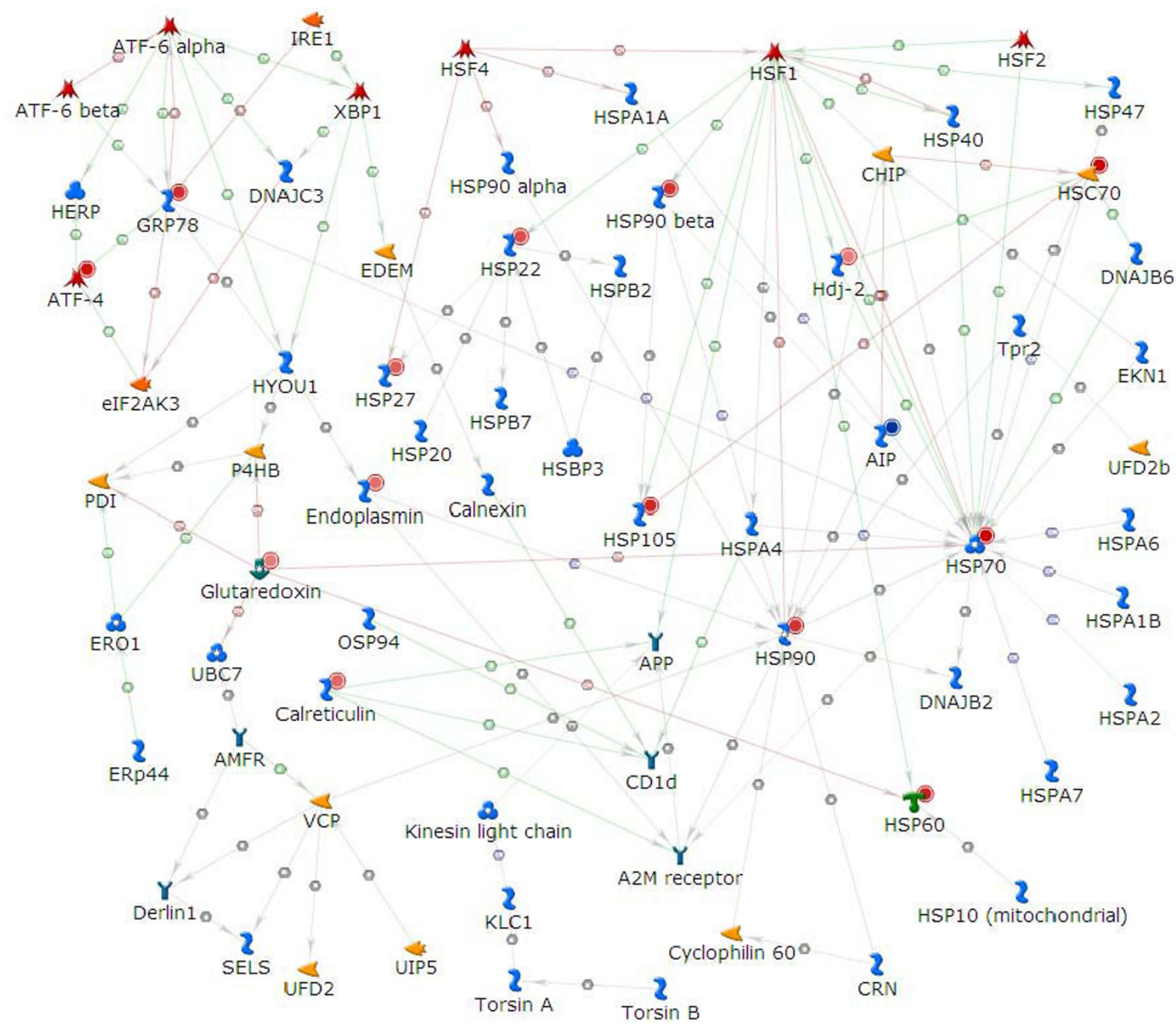

Figure 10

The unfolded-protein response in fasting. The network was generated and linked with available experimental data in the MetaCore ${ }^{\mathrm{TM}}$ suite. Nodes with red or blue circles in top right corner of the network objects, represent up- or down-regulation, respectively, with the shade indicating the intensity of the change. Detailed legend for MC networks is provided in Additional file 3 . 


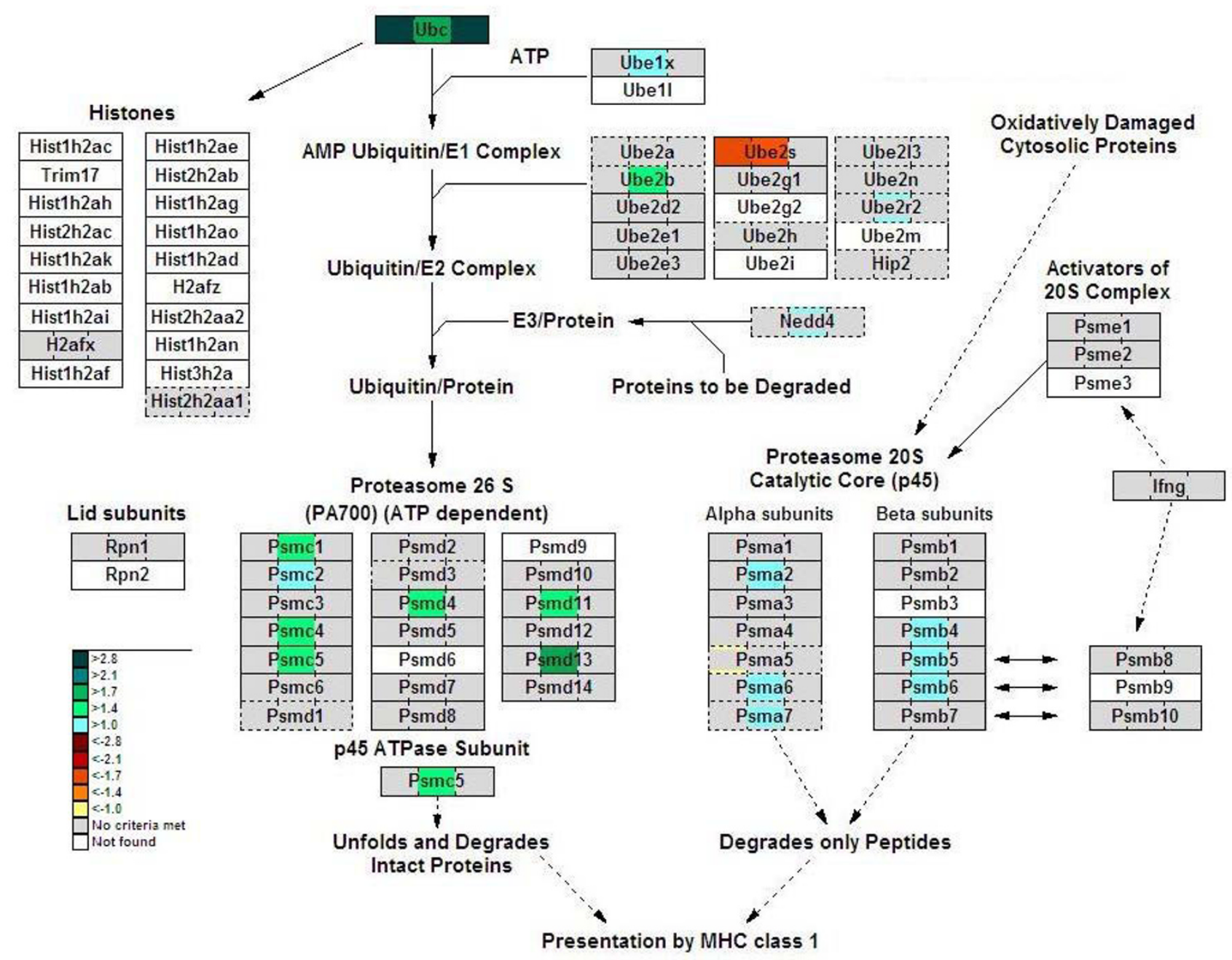

Figure I I

Proteasome degradation in fasting. The colour code of the GenMAPP view is the same as in Figure 5.

quickly resume its homeostatic functions once feeding resumes. Our microarray data show that the adaptive response of the liver to fasting at the level of gene expression is most pronounced during the early phase, with the upregulation of ammonia detoxification persisting up to 72 hours of fasting. Since the technically similar study of Bauer et al. [22] reported enhanced expression of lipidcatabolizing and urea-cycle enzymes after 24 and $48 \mathrm{~h}$ of fasting, the collective data show that the response to fasting in the liver starts already at 12 hours of fasting and becomes maximal between 24 and 48 hours. The hepatic response to total food deprivation, therefore, does not proceed through the global "sugar-fat-protein" sequence that is described for the adaptation on the whole-body level [1-5].

The expression of genes involved in lipid metabolism and ketone-body synthesis, many under PPAR $\alpha$ coordination $[29,26]$, was strongly regulated towards fatty-acid oxida- tion and ketone-body formation. Interestingly, this adaptive response was seen between 12 and 48 hours ([22] and present study) of fasting only, and then faded out. In the rat, this response was recently reported to occur between 3 and 5 days of fasting [30]. Fatty-acid oxidation was accommodated by the identical time frame of the upregulation of the expression of TCA-cycle enzymes and the proteins of the electron-transport chain in response to fasting. The associated oxidative stress and mitochondrial radical formation was apparently sufficiently strong to induce the unfolded-protein (ER stress) response in liver. Thus far, to our knowledge, activation of the unfolded protein response has not been associated with fatty-acid oxidation in the fasting liver, but it is induced by a highfat diet [31]. Similarly, mitochondria in fasting muscle protect themselves against the oxidative stress that results from fat oxidation [32] by accumulating the uncoupling proteins UCP2 and UCP3 [33,34]. 
Table 3: Genes involved in the protein-folding response and oxidative stress that are regulated by fasting

\begin{tabular}{|c|c|c|c|c|}
\hline gene name & gene symbol & $12 \mathrm{~h}$ & $24 \mathrm{~h}$ & $72 \mathrm{~h}$ \\
\hline AH receptor-interacting protein & Aip & -1.78 & -1.63 & \\
\hline Cyclic AMP-dependent transcription factor ATF-4 & Atf4 & & & 2.60 \\
\hline Calreticulin precursor & CalR & & 1.67 & \\
\hline DnaJ homolog subfamily A member I & Dnajal & & 1.40 & \\
\hline Glutaredoxin-I & Glrx & & 1.42 & \\
\hline Heat shock protein HSP $90 \beta$ & Hsp90abl & 2.06 & 2.45 & \\
\hline Endoplasmin precursor & Hsp90bl & & 1.60 & \\
\hline $78 \mathrm{kDa}$ glucose-regulated protein precursor & Hspa5 & 2.31 & 1.86 & \\
\hline Heat-shock cognate $71 \mathrm{kDa}$ protein & Hspa8 & 2.75 & 2.76 & \\
\hline Heat-shock protein $\beta$-I & Hspbl & & 1.76 & \\
\hline Heat-shock protein $\beta-8$ & Hspb8 & & 1.93 & \\
\hline $60 \mathrm{kDa}$ heat-shock protein, mitochondrial precursor & HspdI & & 2.47 & \\
\hline Heat-shock protein 105 kDa & Hsphl & & 2.37 & \\
\hline ubiquitin $\mathbf{C}$ & Ubc & 2.86 & 2.07 & 4.49 \\
\hline ubiquitin-conjugating enzyme E2B & Ube2b & & 1.55 & \\
\hline protease (prosome, macropain) $26 \mathrm{~S}$ subunit, ATPase I & Psmcl & & 1.42 & \\
\hline protease (prosome, macropain) $26 \mathrm{~S}$ subunit, ATPase 4 & Psmc4 & & 1.50 & \\
\hline protease (prosome, macropain) $26 \mathrm{~S}$ subunit, ATPase 5 & Psmc5 & & 1.47 & \\
\hline proteasome (prosome, macropain) $26 \mathrm{~S}$ subunit, non-ATPase, 4 & Psmd4 & & 1.44 & \\
\hline proteasome (prosome, macropain) $26 \mathrm{~S}$ subunit, non-ATPase, il & Psmdl I & & 1.44 & \\
\hline proteasome (prosome, macropain) $26 \mathrm{~S}$ subunit, non-ATPase, 13 & Psmd I 3 & & 2.08 & \\
\hline
\end{tabular}

The role of the liver in gluconeogenesis during fasting is well documented [35-37]. However, the expression of enzymes associated with gluconeogenesis was upregulated only during the first day of fasting and was mainly confined to the malate-aspartate shuttle and Pepck1. In fact, apart from Pepck1, the expression of none of the committed steps in gluconeogenesis was regulated. It is likely that the enhanced expression of TCA-cycle and malateaspartate shuttle enzymes, and the enhanced expression of Pepck1 enhance the flux towards either glucose-6-phosphate or lactate. It is, therefore, remarkable that the expression of glucose-6-phosphatase, a periportal enzyme, and pyruvate kinase, a mainly pericentral enzyme, are not regulated, while the expression of lactate dehydrogenase is only upregulated at 12 and 24 hours. Similarly, the expression and activity of glucose-6-phosphatase in rat liver are upregulated mildly during the first 48 hours of fasting only [38]. Our unpublished data show a similar response in the kidneys of fasting mice, in which Pepck1 is 2-3 fold upregulated at all time points, whereas glucose-6-phosphatase is not regulated. The pronounced accumulation of glycogen in pericentral hepatocytes starting after 24 hours of fasting, which was also observed in 72- and 96-hour fasted rats [39], indicates that pericentral hepatocytes, which do not express glucose-6-phosphatase $[40,41]$, channel glucose-6-phosphate towards glycogen. Since all relevant enzymes are also expressed in periportal hepatocytes, which do not accumulate glycogen, we assume that these hepatocytes contain enough glucose-6phosphatase to produce glucose.

The liver produces $\sim 60 \%$ of the newly produced glucose in starvation, while the kidneys account for 40\% [13]. A recent, but controversial series of experiments suggest that, in addition to the liver [35-37] and kidney [35,42], the small intestine also has the capacity to produce glucose upon prolonged fasting [43,6]. It contributes indirectly, by providing lactate and alanine to the liver in short-term fasting $[17,44]$, and directly by the production of glucose [6] (perhaps up to $27 \%$ of whole-body glucose production in extended fasting in the rat [18]). The concept is controversial, since other studies were unable to detect glucose formation from glutamine in the isolated small intestine of 72 hours fasted rats [45]. Furthermore, the expression of the key gluconeogenic enzyme phosphoenolpyruvate kinase (Pepck1) in the mouse small intestine was reported to amount to only $0.05-1 \%$ of that in the liver after $12 \mathrm{~h}$ hours of fasting [46], also arguing against intestinal gluconeogenesis. We, therefore, compared Pepck mRNA levels in these two organs by qPCR in the fed and 3-days fasting condition (Table 2). While Pepck1 expression in the gut at 12 hours of fasting only amounted to $~ 5 \%$ of that in liver, its expression increased to 18 and $53 \%$ of that in the liver after 24 and 72 hours of fasting, respectively. This finding demonstrates that the issue of intestinal gluconeogenesis during prolonged fasting deserves additional study.

The plasma concentrations of both glucose and lactate remained unchanged during the first 24 hours of fasting, declined temporarily by $35-40 \%$ at 48 hours, and returned to control values between 48 and 72 hours. The maintenance of normal concentrations of glucose and lactate during the first 24 hours of fasting is most likely the result of gluconeogenesis in the liver and kidney. Since the expression of enzymes that are shared by the glycolytic 
and gluconeogenic pathways, declines after 24 hours of fasting, the observed decline at 48 hours may represent a declining contribution of the liver to gluconeogenesis. As we argued earlier, the accumulation of glycogen in the pericentral hepatocytes between 24 and 72 hours of fasting indicates that gluconeogenic intermediates flow towards glucose-6-phosphate and accumulate as glycogen due to the low expression of glucose-6-phosphatase in these hepatocytes. Most likely, a similar or higher flow of gluconeogenic intermediates is present in the periportal hepatocytes, but is exported as glucose due to the high concentration of glucose-6-phosphatese in these cells. Furthermore, the putative production of glucose in the 72hour fasted intestine can also contribute to the circulating glucose level.

The urea-cycle enzymes distinguish themselves from most other genes in the liver in that they were upregulated in expression throughout the period of fasting that was studied. Furthermore, cytosolic glutamate-oxaloacetate transaminase, which mediates the availability of aspartate to the urea-cycle enzyme argininosuccinate synthetase, was also strongly upregulated at 72 hours. Similarly, Oat and Prodh, which supply glutamate to glutamine synthetase for glutamine synthesis in pericentral hepatocytes, were strongly upregulated at all time points studied, but glutamine synthetase itself was not regulated (and even downregulated in another study [22]). Since few aminoacid catabolizing enzymes were upregulated (the exception being the metabolism of sulphur-containing amino acids), most amino-groups were probably carried to the liver as alanine or glutamine, although neither glutamatepyruvate transaminase nor liver glutaminase was upregulated. The coordinate control of ammonia detoxification and the source of ammonia during prolonged fasting therefore deserve attention.

An important question is to what extent we can extrapolate the observations in a small mammal like the mouse to larger animals like humans. The ability to tolerate the absence of food does indeed decline with body size: in the mouse the maximum duration of fasting is 4 days [47], in rat 12-15 days [48], in children 4 weeks and in adult humans 8-9 weeks $[49,13]$. Qualitatively, however, the response to fasting is probably comparable between these mammals, as long as the time scale is adjusted to the size of the animal. Rather than questioning the comparability of small and large animals, our data question whether the implicit extrapolation of the "sugars-fats-proteins" succession of energy substrates during fasting that is based on whole-body energy expenditure $[1,50]$ to individual organs is valid. Microarray studies in rodents that have prospected the adaptive response to fasting of the small intestine [6], liver ([22] and present study), muscle $[23,24,51]$, and a more limited study in kidney focusing on circadian differences in gene expression [52], reveal a different scenario. Muscle and kidney respond to fasting with a progressive change over time in mRNA concentrations of enzymes involved in protein, carbohydrate and fat metabolism. The response in liver peaked at 24-48 hours of fasting in mouse, while most adaptive changes had abated by 72 hours. The intestine, finally, showed an early, but temporary peak of adaptive changes in aminoacid, carbohydrate and fat metabolism at 12 hours of fasting, while a late response, existing almost exclusively of amino-acid catabolizing and gluconeogenic enzymes, gradually developed towards 72 hours of fasting. These differences in pattern and amplitude of gene expression change in different organs can be used to look for circulating biomarkers that reflect the functions of organs during adaptive responses.

\section{Conclusion}

Based on whole-body energy expenditure, the "sugarsfats-proteins" sequence of energy substrates during fasting was suggested. In our extensive microarray studies of the response to fasting in the gut [6] and liver (present study), we found no support for this intuitively attractive model at the individual organ level. The liver markedly differed from the biphasic response pattern in the small intestine (with peaks at 12 and 72 hours) in that its adaptive response peaked at 24-48 hours of fasting, while most adaptive changes had abated by 72 hours. Expression profiling and pathway analysis revealed that genes involved in amino-acid, lipid, carbohydrate and energy metabolism responded most significantly to fasting, with no temporal separation between them. Even though the liver lost $50 \%$ of its initial weight during 3 days of fasting, its basic morphology remained preserved, showing that the liver can quickly resume its homeostatic functions when feeding resumes.

\section{Methods}

\section{Animals and tissues}

Livers were harvested from the same mice that were used to study the effects of fasting on the small intestine [6]. Briefly, 6 week-old male FVB mice (Charles River, Maastricht, The Netherlands) were fasted for $0,12,24$, or 72 hours before sacrifice ( $\mathrm{N} \geq 8$ per group). The animals were kept in metabolic cages to prevent the consumption of bedding and were kept warm with an infrared lamp. Body weight was determined daily. The daily rate of body or organ mass loss was calculated as described [53]. The animals were sacrificed between 9:00 and 10:00 a.m. by cervical dislocation. The liver was isolated quickly, freed from the gall bladder, cut into pieces and either snap-frozen in liquid $\mathrm{N}_{2}$ and stored at $-80^{\circ} \mathrm{C}$, or fixed overnight at $4{ }^{\circ} \mathrm{C}$ in $4 \%$ buffered formaldehyde or a mixture of methanol, acetone, and water (2:2:1 by volume). The study followed the Dutch guidelines for the use of experimental animals and was approved by the AMC Animal Experiments Committee. 


\section{RNA isolation and quantification}

Total liver RNA was extracted from frozen tissue with TRIzol reagent (Invitrogen, Breda, The Netherlands). The RNA quality was assessed using the RNA 6000 Nano LabChip $^{\circledast}$ Kit in an Agilent 2100 bioanalyzer (Agilent Technologies, Palo Alto, USA). Additional mRNA quantification for Cps (not present on the microarray) and the qPCR validation of the changes derived from the microarray read-outs was performed as described [54] (Supplementary Table 2, Additional file 1). The gene-specific primer sequences are shown in Supplementary Table 3 (Additional file 1). mRNA concentration was calculated using the LinReg program [55]. The significance of the qPCR data was assessed by Student's t-test.

\section{Microarrays}

Three microarrays (Mouse Development Oligo Microarrays G4120A; $22 \mathrm{~K}$; Agilent) per experimental condition and a robust reference design were used [56]. Per microarray, $20 \mu \mathrm{g}$ mRNA, pooled from 2 livers, was reverse transcribed with Cy3-labelled dCTP (Perkin Elmer, Boston, USA), using the Agilent Fluorescent Direct Label Kit. Cy5labeled cDNA, produced from RNA pooled from the livers of 6 fed animals, served as the common reference across all arrays. Hybridized cDNAs were detected with Agilent's dual-laser microarray-slide scanner and the data retrieved with Agilent's Feature Extraction software 6.1.1. The data discussed in this publication have been deposited in NCBIs Gene Expression Omnibus (GEO; [66]) and are accessible through GEO Series accession number GSE10653.

\section{Data analysis}

The data were processed and analyzed as described [6]. In brief, background-subtracted intensities were calculated using foreground and background median signals, and normalized with the quantile normalization method. An ANOVA model was applied to the common reference channel only, to remove outliers and local artefacts, and detect non-uniform hybridization [57]. Differentially expressed genes were identified with the Split-Factor ANOVA directly by comparing the green (experimental) and red (reference) signals, and indirectly, across-arrays, by comparing the Cy3 signals of starved and fed animals. A consensus between the direct and across-array ANOVA ensures that the final results do not suffer from either dyegene effects or array-specific noise. Genes that received a concordant significance call in 2 out of 3 microarrays $(\mathrm{P}<$ 0.01 ) from both the direct and across-array split-factor ANOVA were taken into further consideration. Given the high sensitivity of Agilent arrays [58], we opted for 1.4fold change as inclusion criterion for a gene.

To perform cluster analysis, Pearson correlation was set as distance measure and complete linkage as agglomeration method. The normalized log-ratio (Cy5/Cy3) expression values of the top 500 most differentially expressed genes between fasted and normal fed mice were used to calculate the correlation between samples. R/Bioconductor [59] was used to create the clusters.

Pathway, network and gene-set enrichment analyses were applied system-wide, using the MetaCore ${ }^{\mathrm{TM}}$ suit (GeneGo, Inc., St. Joseph, MI, USA) [60,61]. The significance of changes in expression in pathways or networks is based on the degree of overlap between the user's dataset and a set of genes corresponding to a network or pathway queried. The problem is cast as the probability that a randomly obtained overlap of a certain size between the user's set and a network/pathway follows a hypergeometric distribution. Additionally, pathway analysis and visualisation was performed using GenMAPP [62] (2.0 $\beta$ version) software (Gladstone Institutes, UCSF, San Francisco, USA). In all applications, $\mathrm{P}<0.01$ and $\geq 1$.4-fold change were used as inclusion criteria.

To assess the significance of the results other than microarray data, ANOVA and Student's t-test were employed. The error bars in the figures represent the standard error of the mean (SEM).

\section{Histology and immunohistochemistry}

Sections were stained with hematoxylin and azophloxine, or immunohistochemically as described [6,63]. Monoclonal anti-glutamine synthetase (Transduction Laboratories, Lexington, KY) and polyclonal anti-carbamoyl phosphate synthetase (CPS, [64]) antibodies were used. Antibody binding was visualized with goat anti-mouse or goat anti-rabbit IgG, both coupled to alkaline phosphatase (Sigma).

Periodic acid-Schiff (PAS) staining was performed to visualize glycogen in the liver. The sections were incubated for 30 minutes in $0.5 \%$ periodic acid, followed by incubation in Schiff's reagent for 30 minutes, and counterstained in haematoxylin for 6 minutes. The identity of glycogen was verified by predigesting a serial section with $0.1 \% \alpha$-amylase for 45 minutes prior to staining.

\section{Biochemical measurements}

Blood ammonia levels were determined immediately after collecting blood from the caval vein, using Ammonia Checker II (model AA-4120, Kyoto Daiichi Kagaku Co., Japan) and the corresponding Ammonia test kit II (Arkray, Inc., Japan).

For determination of free amino acids, $10 \mu \mathrm{L}$ of plasma (blood was collected in heparin-containing tubes, and centrifuged for $5 \mathrm{~min}$ at $14,000 \mathrm{rpm}$ at $4{ }^{\circ} \mathrm{C}$ ) was mixed with $0.8 \mathrm{mg}$ of lyophilized sulphosalicylic acid, centrifuged, and the supernatant stored at $-80^{\circ} \mathrm{C}$. Amino-acid analysis was performed using a gradient reverse-phase 
HPLC system, with precolumn derivatization with o-phtaladehyde (Pierce) and 3-mercaptopropionic acid (Sigma), and fluorescence detection [65]. Separation was performed using an Omnisphere 3 column (Varian, Middelburg, The Netherlands).

For glucose and lactate measurements, plasma was acidified with 1 volume $2 \mathrm{M}(12 \%)$ perchloric acid, centrifuged for 15 minutes, and neutralized with $1 \mathrm{M} \mathrm{MES} / 2 \mathrm{M} \mathrm{KOH}$. Plasma glucose and lactate concentrations were measured enzymatically, using the NOVOstar reader (BMG Labtech GmbH, Offenburg, Germany).

\section{Abbreviations}

Acaa1: acetyl-coenzyme A acyltransferase $1 ;$ Acad/- $v,-l,-m,-$ sh: acyl-coenzyme A dehydrogenase/very long, long, medium and short chain; Acaddm: acetyl-coenzyme A dehydrogenase, medium chain; Aco2: aconitase 2; Aip: $\mathrm{AH}$ receptor-interacting protein; Aldoa: aldolase $1 \mathrm{~A}$ isoform; ANOVA: analysis of variance; Asl, Arly: argininosuccinate lyase; Ass1, Assy: argininosuccinate synthetase 1; Atf4: Cyclic AMP-dependent transcription factor ATF-4 Atp5a1: ATP synthase subunit $\alpha$; Atp5d: ATP synthase $\delta$ chain; Atp5g1: ATP synthase lipid-binding protein; Cac, Slc25a20: carnitine/acylcarnitine fatty-acid translocase; Calr: calreticulin precursor; Cat: catalase; Cps: carbamoylphosphate synthetase; Cpt2: carnitine palmitoyltransferase 2; Cy3, Cy5: fluorescent dyes of the cyanine dye family; Dlst: dihydrolipoamide S-succinyltransferase; Dnaja1: DnaJ homolog subfamily A member 1; Echdc3: enoyl coenzyme A hydratase domain containing 3; Egr1: early growth response protein 1; Ehhadh: enoyl-coenzyme A, hydratase/3-hydroxyacyl-coenzyme; Eno1: enolase 1 $\alpha$; ER: endoplasmic reticulum; Fh1: fumarate hydratase 1; FVB: mouse strain sensitive to Friend leukaemia virus B; Gapdh: glyceraldehyde-3-phosphate dehydrogenase; Glrx: glutaredoxin-1; Got1: glutamate-oxaloacetate transaminase cytosolic; Got2: glutamate-oxaloacetate transaminase mitochondrial; Gpi1: glucosephosphate isomerase 1; GS, Glns, Glul: glutamine synthetase; HA: hematoxylin, azophloxin; Hadhb-trifunctional protein, $\beta$-subunit; Hadhsc: hydroxyacyl-coenzyme A dehydrogenase, short chain; Hmgcl: 3-hydroxy-3-methylglutaryl-coenzyme A lyase; Hmgcs2: 3-hydroxy-3-methylglutaryl-Coenzyme A synthase 2; Hsp90ab1: heat shock protein HSP 90-beta; Hsp90b1: endoplasmin precursor; Hspa5: 78 kDa glucoseregulated protein precursor; $H s p a 8$ : heat shock cognate 71 kDa protein; Hspb1: heat-shock protein beta-1; Hspb8: heat-shock protein beta-8; $H s p d v$ : $160 \mathrm{kDa}$ heat shock protein, mitochondrial precursor; Hsph1: heat-shock protein $105 \mathrm{kDa}$; Idh3b: isocitrate dehydrogenase $3 \beta$ (NAD+); KOH: potassium hydroxide; Mapk14: mitogen-activated protein kinase 14; Mdh1: malate dehydrogenase 1; MES: 2-(N-Morpholino)ethanesulfonic acid; Ndufa10: NADH dehydrogenase [ubiquinone] $1 \alpha$ subcomplex subunit 10;
Ndufv: NADH dehydrogenase [ubiquinone] flavoprotein; Oat: ornithine aminotransferase; Ogdh: oxoglutarate dehydrogenase; PAS: Periodic acid-Schiff; Pepck, Pck1: phosphoenolpyruvate carboxykinase 1; Pgam1: phosphoglycerate mutase 1; Ppar $\alpha$ : peroxisome proliferatoractivated receptor, $\alpha$ isotype; Prodh: proline dehydrogenase; Psmc: protease (prosome, macropain) $26 \mathrm{~S}$ subunit, ATPase; Psmd: proteasome (prosome, macropain) $26 \mathrm{~S}$ subunit, non-ATPase; qPCR: quantitative polymerase chain reaction; RNA: ribonucleic acid; Scd1: stearoylCoenzyme A desaturase 1; Sod1: superoxide dismutase; TCA: tricarboxylic acid cycle; Tpi1: triosephosphate isomerase 1 ; UbC: ubiquitin C; Ube2b: ubiquitin-conjugating enzyme E2B; Ucp2: uncoupling protein 2; Uqcrc1: ubiquinol-cytochrome-c reductase complex core protein 1

\section{Authors' contributions}

MS carried out the biological part of the study and prepared the manuscript. AS contributed to the biological part of the research. DdW performed a part of biochemical measurements. DW designed and carried out a part of the bioinformatics analysis of the data, together with LGP and EVLvT. AvK supervised this part of the study. YN supported the data analysis in the MetaCore suite. TH and WL supervised the biological part of the study.

\section{Additional material}

\section{Additional file 1}

Supplementary tables. Supplementary table 1 shows amino-acid concentrations in plasma after 0,12, 24, 48 and 72 hours of fasting. Supplementary table 2 shows $q P C R$ validation of microarray data.

Supplementary table 3 contains gene-specific primer sequences, product lengths and annealing temperatures.

Click here for file

[http://www.biomedcentral.com/content/supplementary/14712164-9-528-S1.doc]

\section{Additional file 2}

Fold changes in liver response to fasting. The file contains lists of all the genes significantly regulated in the liver ( $\geq 1.4$ fold) per time point of fasting.

Click here for file

[http://www.biomedcentral.com/content/supplementary/14712164-9-528-S2.xls]

\section{Additional file 3}

MetaCore legend. The file contains a legend for the pathways and the network created in MetaCore suite shown in the Figures 7, 8 and 10. Click here for file

[http://www.biomedcentral.com/content/supplementary/14712164-9-528-S3.pdf]

\section{Acknowledgements}

This work was supported by the Dutch Ministry of Economic Affairs through the Innovative Oriented Research Program on Genomics (IOP 
Genomics: IGE0I016). EVLvT was supported by the FP6 European Union Project 'Peroxisome' LSHG-CT-2004-512018.

\section{References}

I. Le Maho Y, Vu Van Kha H, Koubi H, Dewasmes G, Girard J, Ferre P, Cagnard M: Body composition, energy expenditure, and plasma metabolites in long-term fasting geese. Am J Physio |98|, 24 |(5):E342-E354.

2. Cherel Y, Attaix D, Rosolowska-Huszcz D, Belkhou R, Robin JP, Arna $M$, Le MY: Whole-body and tissue protein synthesis during brief and prolonged fasting in the rat. Clin Sci (Lond) 1991, 8I:6II-6I9.

3. Cherel Y, Le MY: Refeeding after the late increase in nitrogen excretion during prolonged fasting in the rat. Physiol Behav I991, 50:345-349.

4. Belkhou R, Cherel Y, Heitz A, Robin J, Le Maho Y: Energy contribution of proteins and lipids during prolonged fasting in the rat. Nutrition Research 1991, I I:365-374.

5. Habold C, Chevalier C, Dunel-Erb S, Foltzer-Jourdainne C, Le Maho $\mathrm{Y}$, Lignot $\mathrm{JH}$ : Effects of fasting and refeeding on jejunal morphology and cellular activity in rats in relation to depletion of body stores. Scandinavian Journal of Gastroenterology 2004, 39:531-539.

6. Sokolovic M, Wehkamp D, Sokolovic A, Vermeulen J, Gilhuijs-Pederson LA, van Haaften RI, Nikolsky $Y$, Evelo $C T$, van Kampen $A H$, Hakvoort TB, Lamers WH: Fasting induces a biphasic adaptive metabolic response in murine small intestine. BMC Genomics 2007, 8:36I.

7. Habold C, Foltzer-Jourdainne C, Le Maho Y, Lignot JH, Oudart H: Intestinal gluconeogenesis and glucose transport according to body fuel availability in rats. J Physiol (Lond) 2005, 566:575-586.

8. Dou Y, Gregersen S, Zhao J, Zhuang F, Gregersen H: Morphometric and biomechanical intestinal remodeling induced by fasting in rats. Dig Dis Sci 2002, 47: I I58- I 168.

9. Johnstone AM, Faber P, Gibney ER, Elia M, Horgan G, Golden BE, Stubbs RJ: Effect of an acute fast on energy compensation and feeding behaviour in lean men and women. Int J Obes Relat Metab Disord 2002, 26: 1623-1628.

10. Owen OE, Reichard GA Jr, Patel MS, Boden G: Energy metabolism in feasting and fasting. Adv Exp Med Biol 1979, I I I:169-188.

II. Corssmit EP, Romijn JA, Sauerwein HP: Regulation of glucose production with special attention to nonclassical regulatory mechanisms: a review. Metabolism 200I, 50:742-755.

12. Johnstone AM: Fasting - the ultimate diet? Obes Rev 2007, 8:2II-222

13. Cahill GF Jr: Fuel metabolism in starvation. Annu Rev Nutr 2006 26: $1-22$.

14. Owen OE, Smalley KJ, D'Alessio DA, Mozzoli MA, Dawson EK: Protein, fat, and carbohydrate requirements during starvation: anaplerosis and cataplerosis. Am J Clin Nutr 1998, 68: 12-34.

15. Marliss EB, Aoki TT, Pozefsky T, Most AS, Cahill GF Jr: Muscle and splanchnic glutamine and glutamate metabolism in postabsorptive and starved man. I Clin Invest 1971, 50:814-817.

16. Felig P, Owen O, Wahren J, Cahill G): Amino acid metabolism during prolonged starvation. J Clin Invest 1969, 48:584-594.

17. Windmueller HG, Spaeth AE: Identification of ketone bodies and glutamine as the major respiratory fuels in vivo for postabsorptive rat small intestine. J Biol Chem 1978, 253:69-76.

18. Mithieux G, Gautier-Stein A, Rajas F, Zitoun C: Contribution of intestine and kidney to glucose fluxes in different nutritional states in rat. Comparative Biochemistry and Physiology B-Biochemistry \& Molecular Biology 2006, |43:|95-200.

19. Goodman AD, Fuisz RE, Cahill GF Jr: Renal gluconeogenesis in acidosis, alkalosis, and potassium deficiency: its possible role in regulation of renal ammonia production. J Clin Invest 1966 , 45:612-619.

20. Norrelund $\mathrm{H}$ : The metabolic role of growth hormone in humans with particular reference to fasting. Growth Hormone \& IGF Research 2005, I 5:95-122.

21. Owen OE, Morgan AP, Kemp HG, Sullivan JM, Herrera MG, Cahill GF Jr: Brain metabolism during fasting. I Clin Invest 1967, 46: $1589-1595$

22. Bauer M, Hamm AC, Bonaus M, Jacob A, Jaekel J, Schorle H, Pankratz $\mathrm{MJ}$, Katzenberger JD: Starvation response in mouse liver shows strong correlation with life-span-prolonging processes. Physiol Genomics 2004, I 7:230-244

23. Jagoe RT, Lecker SH, Gomes M, Goldberg AL: Patterns of gene expression in atrophying skeletal muscles: response to food deprivation. FASEB J 2002, 16:1697-17/2.

24. Lecker SH, Jagoe RT, Gilbert A, Gomes M, Baracos V, Bailey J, Price SR, Mitch WE, Goldberg AL: Multiple types of skeletal muscle atrophy involve a common program of changes in gene expression. Faseb Journal 2004, |8:39-5|

25. Burg MB: Molecular basis of osmotic regulation. Am J Physiol 1995, 268:F983-F996.

26. Goldstein JL, Brown MS: Regulation of the mevalonate pathway. Nature 1990, 343:425-430.

27. Minc E, de CP, Masson P, Thiery L, Dutertre S, mor-Gueret M, Jaulin $C$ : The human copper-zinc superoxide dismutase gene (SODI) proximal promoter is regulated by SpI, Egr-I, and WTI via non-canonical binding sites. J Biol Chem 1999, 274:503-509.

28. Formigari A, Irato $P$, Santon A: Zinc, antioxidant systems and metallothionein in metal mediated-apoptosis: biochemical and cytochemical aspects. Comp Biochem Physiol C Toxicol Pharmacol 2007, I 46:443-459.

29. Leone TC, Weinheimer CJ, Kelly DP: A critical role for the peroxisome proliferator-activated receptor alpha (PPARalpha) in the cellular fasting response: the PPARalpha-null mouse as a model of fatty acid oxidation disorders. Proc Natl Acad $\mathrm{Sci}$ USA 1999, 96:7473-7478.

30. Li RY, Zhang QH, Liu Z, Qiao J, Zhao SX, Shao L, Xiao HS, Chen JL, Chen MD, Song HD: Effect of short-term and long-term fasting on transcriptional regulation of metabolic genes in rat tissues. Biochem Biophys Res Commun 2006, 344:562-570.

31. Eizirik DL, Cardozo AK, Cnop M: The role for endoplasmic reticulum stress in diabetes mellitus. Endocr Rev 2008, 29:42-6I.

32. Schrauwen $P$, Hesselink MK: The role of uncoupling protein 3 in fatty acid metabolism: protection against lipotoxicity? Proc Nutr Soc 2004, 63:287-292.

33. Samec S, Seydoux J, Dulloo AG: Role of UCP homologues in skeletal muscles and brown adipose tissue: mediators of thermogenesis or regulators of lipids as fuel substrate? FASEB J 1998 | 2:7|5-724.

34. Cadenas S, Buckingham JA, Samec S, Seydoux J, Din N, Dulloo AG, Brand MD: UCP2 and UCP3 rise in starved rat skeletal muscle but mitochondrial proton conductance is unchanged. FEBS Lett 1999, 462:257-260.

35. Owen OE, Felig P, Morgan AP, Wahren J, Cahill GF Jr: Liver and kidney metabolism during prolonged starvation. J Clin Invest 1969 , 48:574-583.

36. Klover PJ, Mooney RA: Hepatocytes: critical for glucose homeostasis. Int J Biochem Cell Biol 2004, 36:753-758.

37. Wahren J, Ekberg K: Splanchnic regulation of glucose production. Annu Rev Nutr 2007, 27:329-345.

38. Minassian C, Zitoun C, Mithieux G: Differential time course of liver and kidney glucose-6 phosphatase activity during longterm fasting in rat correlates with differential time course of messenger RNA level. Mol Cell Biochem 1996, I 55:37-4I.

39. Minassian C, Ajzannay A, Riou JP, Mithieux G: Investigation of the mechanism of glycogen rebound in the liver of 72- hour fasted rats. J Biol Chem 1994, 269: I6585-16588.

40. Jungermann K, Heilbronn R, Katz N, Sasse D: The glucose/glucose6-phosphate cycle in the periportal and perivenous zone of rat liver. Eur J Biochem 1982, 1 23:429-436.

4I. Jonges GN, Van Noorden C], Lamers WH: In situ kinetic parameters of glucose-6-phosphatase in the rat liver lobulus. J Biol Chem 1992, 267:4878-488।.

42. Conjard A, Brun V, Martin M, Baverel G, Ferrier B: Effect of starvation on glutamine ammoniagenesis and gluconeogenesis in isolated mouse kidney tubules. Biochemical Journal 2002 368:30I-308.

43. Mithieux G: The new functions of the gut in the control of glucose homeostasis. Curr Opin Clin Nutr Metab Care 2005 8(4):445-449.

44. Watford M: Glutamine-Metabolism in Rat Small-Intestine Synthesis of 3-Carbon Products in Isolated Enterocytes. Biochimica et Biophysica Acta-General Subjects 1994, I 200:73-78.

45. Martin G, Ferrier B, Conjard A, Martin M, Nazaret R, Boghossian M, Saade F, Mancuso C, Durozard D, Baverel G: Glutamine glucone- 
ogenesis in the small intestine of $72 \mathrm{~h}$-fasted adult rats is undetectable. Biochem J 2007, 40 I:465-473.

46. Azzout-Marniche D, Gaudichon C, Blouet C, Bos C, Mathe V, Huneau JF, Tome D: Liver glyconeogenesis: a pathway to cope with postprandial amino acid excess in high-protein fed rats? Am J Physiol Regul Integr Comp Physiol 2007, 292:R I 400-R I 407.

47. Cuendet GS, Loten EG, Cameron DP, Renold AE, Marliss EB: Hormone-substrate responses to total fasting in lean and obese mice. Am J Physiol 1975, 228:276-283

48. Koubi H, Robin J, Dewasmes G, Le Maho Y, Frutoso J, Robin J: Fasting-induced rise in locomotor activity in rats coincides with increased protein utilization. Physiology and Behaviour I991, 50:337-343.

49. Leiter L, Marliss E: Survival during fasting may depend on fat as well as protein stores. JAMA 1982, 248:2306-2307.

50. Caloin M: Modeling of lipid and protein depletion during total starvation. Am J Physiol Endocrinol Metab 2004, 287:E790-E798.

5I. Xiao XQ, Grove KL, Smith MS: Metabolic Adaptations in Skeletal Muscle during Lactation: Complementary Deoxyribonucleic Acid Microarray and Real-Time Polymerase Chain Reaction Analysis of Gene Expression. Endocrinology 2004 1 45:5344-5354.

52. Kita Y, Shiozawa M, Jin W, Majewski RR, Besharse JC, Greene AS, Jacob HJ: Implications of circadian gene expression in kidney, liver and the effects of fasting on pharmacogenomic studies. Pharmacogenetics 2002, I 2:55-65.

53. Habold C, Foltzer-Jourdainne C, Le Maho Y, Lignot JH: Intestinal apoptotic changes linked to metabolic status in fasted and refed rats. Pflugers Archiv-European Journal of Physiology 2006 45 I:749-759.

54. Lekanne Deprez RH, Fijnvandraat AC, Ruijter JM, Moorman AF: Sensitivity and accuracy of quantitative real-time polymerase chain reaction using SYBR green I depends on CDNA synthesis conditions. Anal Biochem 2002, 307:63-69.

55. Ramakers C, Ruijter JM, Deprez RHL, Moorman AFM: Assumptionfree analysis of quantitative real-time polymerase chain reaction (PCR) data. Neuroscience Letters 2003, 339:62-66.

56. Dobbin K, Simon R: Comparison of microarray designs for class comparison and class discovery. Bioinformatics 2002, I 8: |438-| 445 .

57. Gilhuijs-Pederson LA, van Kampen AHC, AMC/UvA: Analysis of a microarray data set. Patent, PCT/EP2004/006245 2004

58. Hughes TR, Mao M, Jones AR, Burchard J, Marton MJ, Shannon KW, Lefkowitz SM, Ziman M, Schelter JM, Meyer MR, Kobayashi S, Davis C. Dai HY, He YDD, Stephaniants SB, Cavet G, Walker WL, West A, Coffey E, Shoemaker DD, Stoughton R, Blanchard AP, Friend SH, Linsley PS: Expression profiling using microarrays fabricated by an ink-jet oligonucleotide synthesizer. Nature Biotechnology 200I, 19:342-347.

59. Gentleman RC, Carey VJ, Bates DM, Bolstad B, Dettling M, Dudoit S, Ellis B, Gautier L, Ge Y, Gentry J, Hornik K, Hothorn T, Huber W, lacus S, Irizarry R, Leisch F, Li C, Maechler M, Rossini AJ, Sawitzki G, Smith C, Smyth G, Tierney L, Yang JY, Zhang J: Bioconductor: open software development for computational biology and bioinformatics. Genome Biol 2004, 5:R80.

60. Ekins S, Kirillov E, Rakhmatulin EA, Nikolskaya T: A novel method for visualizing nuclear hormone receptor networks relevant to drug metabolism. Drug Metabolism and Disposition 2005 , 33:474-48I.

61. Nikolsky Y, Nikolskaya T, Bugrim A: Biological networks and analysis of experimental data in drug discovery. Drug Discovery Today 2005, 1 0:653-662.

62. Dahlquist KD, Salomonis N, Vranizan K, Lawlor SC, Conklin BR: GenMAPP, a new tool for viewing and analyzing microarray data on biological pathways. Nat Genet 2002, 3I:19-20.

63. Lenaerts K, Sokolovic M, Bouwman FG, Lamers WH, Mariman EC Renes J: Starvation induces phase-specific changes in the proteome of mouse small intestine. Journal of Proteome Research 2006, 5:2113-2122.

64. Charles R, De GA, Lamers WH, Moorman AF: Control of the changes in rat-liver carbamoyl-phosphate synthase (ammonia) protein levels during ontogenesis: evidence for a perinatal change in immunoreactivity of the enzyme. Mech Ageing Dev 1983, 22: 193-203.
65. van Eijk HM, Heijden MA van der, van Berlo CL, Soeters PB: Fully automated liquid-chromatographic determination of amino acids. Clin Chem 1988, 34:2510-2513.

66. Gene Expression Omnibus [http://www.ncbi.nlm.nih.gov/geo]
Publish with Biomed Central and every scientist can read your work free of charge

"BioMed Central will be the most significant development for disseminating the results of biomedical research in our lifetime. "

Sir Paul Nurse, Cancer Research UK

Your research papers will be:

- available free of charge to the entire biomedical community

- peer reviewed and published immediately upon acceptance

- cited in PubMed and archived on PubMed Central

- yours - you keep the copyright
BioMedcentral 